\title{
Investigation of the base flow of a generic space launcher with dual-bell nozzle
}

\author{
Sven Scharnowski ${ }^{1}$ (I) $\cdot$ Christian J. Kähler ${ }^{1}$
}

Received: 5 May 2020 / Revised: 6 August 2020 / Accepted: 24 August 2020 / Published online: 19 September 2020

(C) The Author(s) 2020

\begin{abstract}
The typical afterbody flow of a space launcher is characterized by a strong interaction of the engine's exhaust jet and the separated shear layer emerging from the main body. This interaction is further complicated by strong changes in the spatial and temporal behavior of the afterbody flow during the atmospheric ascent of a launcher. Theoretically, a dual-bell nozzle not only allows for a gain in payload compared to standard single-bell nozzles, but also it alters the wake flow topology due to the two nozzle modes. To predict the benefits as well as the additional risks, the afterbody flow of a generic space launcher model equipped with a cold-flow dual-bell nozzle is investigated in detail. The flow was analyzed for sub-, trans- and supersonic Mach numbers ranging from 0.3 to 2.9 for a variety of nozzle pressure ratios. Particle image velocimetry measurements and schlieren measurements with high repetition rate were performed to determine the dynamics of the separated shear layer, the nozzle jet and their interaction. It is shown that the reattachment length of the base flow decreases with increasing nozzle pressure ratio. Furthermore, the nozzle pressure ratio at which the dual-bell nozzle switches from sea-level mode to altitude mode is reduced by $15 \%$ with high subsonic outer flow and by as much as $65 \%$ for an outer flow at a Mach number of 1.6. Even for a constant nozzle pressure ratio, the nozzle flow topology depends on the Mach number of the outer flow.
\end{abstract}

keywords Nozzle flow $\cdot$ Dual bell $\cdot$ Flow separation

\section{Introduction}

Access to space is of great importance for the security and comfort of our lives. Satellites in various orbits around the Earth are used to navigate, to communicate and to forecast the weather, among other things. At present, there are about 5000 satellites orbiting the earth and every year hundreds more are added, with an upward trend ${ }^{1}$. Currently, space launchers are our only means of reaching outer space. Therefore, they are absolutely essential for transporting the satellites that we need for such a wide variety of purposes. This drives the need to develop launch vehicles that are as safe

Electronic supplementary material The online version of this article (https://doi.org/10.1007/s12567-020-00333-5) contains supplementary material, which is available to authorized users.

Sven Scharnowski

sven.scharnowski@unibw.de

1 Bundeswehr University Munich, Institute of Fluid Mechnics and Aerodynamics, Neubiberg, Germany and efficient as possible from an economic and ecological point of view.

The interaction between the external flow and the structure in the afterbody of a launch vehicle is considered a critical factor. In recent years, many experiments $[4,5,11$, $15,18,31,42,43,49]$ and computational flow simulations $[10,23,28,48,54-56]$ have focused on the dynamics of the separated / reattached shear layer coming from the end of the main body of a space launcher model. It was shown that the shear layer vortices impinge on the rear end of nozzle fairing for a generic Ariane 5-like geometry. This reattachment causes strong wall pressure fluctuations which lead to increased dynamic loads for the nozzle structure. The loads are strongest in the transonic range and the characteristic frequencies of the pressure fluctuations are likely to interfere with the structural modes of the nozzle, resulting in so-called buffeting. It is, therefore, very important to also consider the external flow when carrying out tests with new nozzle concepts.

During the atmospheric ascent, a space launcher is exposed to widely varying ambient pressures, which strongly

\footnotetext{
${ }_{1}^{1}$ https://www.pixalytics.com/satellites-orbiting-earth-2019/.
} 

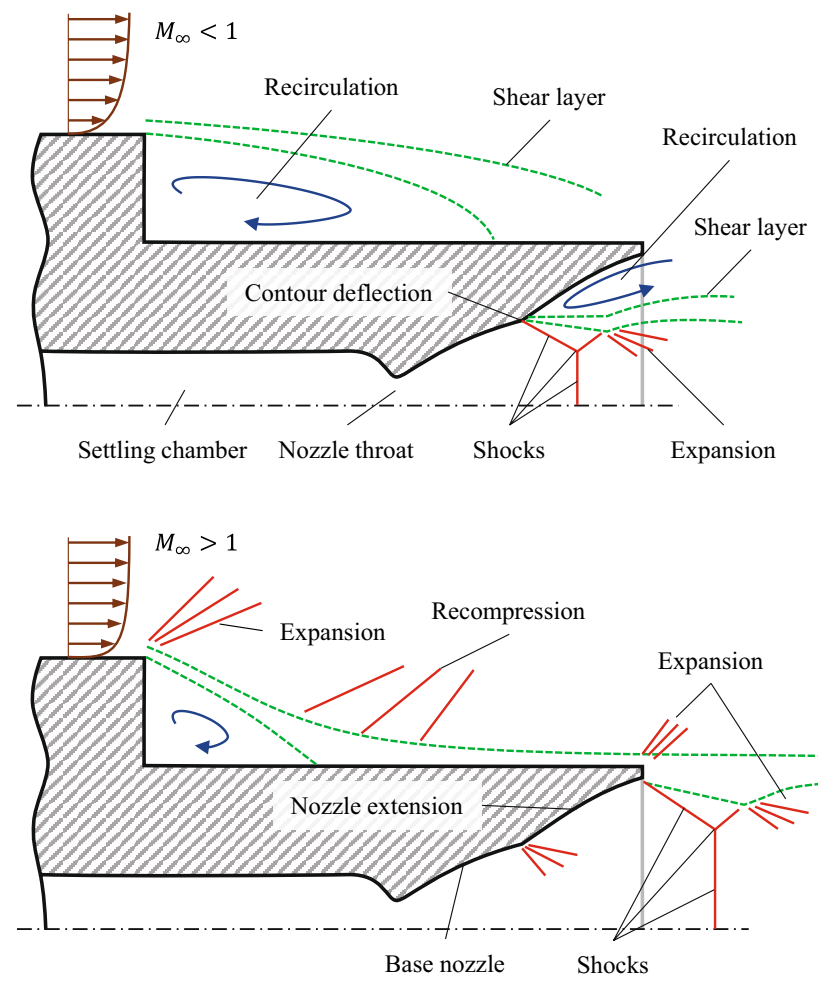

Fig. 1 Sketch of a generic space launcher afterbody with dual-bell nozzle and occurring flow phenomena. Top: Subsonic outer flow and sea-level nozzle mode. Bottom: Supersonic outer flow with altitude nozzle mode

influences the efficiency of the thrust nozzles used for propulsion. Single-bell nozzle shapes like the truncated ideal contour (TIC) or the thrust optimized contour (TOC) are widely used in space activities. However, they have optimum performance only for the design conditions, i.e., a certain flight altitude. However, they only achieve optimum performance when the design conditions are met, e.g., at a certain flight altitude. Below and above this altitude, losses occur due to the fact that the area ratio between nozzle exit and nozzle throat does not correspond to the pressure conditions needed for an adapted nozzle flow.

To increase the performance of space launch vehicles, altitude-adaptive nozzles offer a high potential. However, this is often accompanied by increased complexity, which could lead to an increased risk of failure. Hagemann et al. [16] discussed in detail the possible gain and the risk of altitude-adaptive nozzles like the dual-bell nozzle, other nozzles with devices for forced flow separation, plug nozzles, expansion-deflection nozzles and extendible nozzles. The dual-bell nozzle, first introduced by [12], is a promising concept which provides a one-step adaption without any moving parts. The dual-bell concept consists of a base nozzle, which transitions into a nozzle extension with a contour deflection, see Fig. 1. At low altitude, the exhaust jet separates at the contour deflection resulting in a small area ratio between exit and throat of the nozzle. When the ambient pressure drops sufficiently, the second bell is also filled and the area ratio rises suddenly. Stark et al. [46] showed that the dual-bell nozzle concept could increase the payload of European's launcher Ariane 5 by up to $490 \mathrm{~kg}$, which is a gain of about $5 \%$.

Although the dual-bell nozzle seems to be the most promising choice among the adaptive nozzles, there is also an increased risk associated with it. One important point in the risk assessment is the transition from the so-called sea-level mode (top in Fig. 1) to the altitude mode (bottom in Fig. 1), in which the flow separation moves from the contour deflection towards the nozzle exit. This transition causes strong mechanical loads for a short time period. To minimize the loads, it is important that the transition from sea-level to altitude mode only happens once without any re-transition. To reduce the chance of re-transition, a large hysteresis between the pressure for transition and re-transition can be achieved by a positive pressure gradient nozzle extension $[13,26]$. Additionally, a large extension length leads to a more stable transition, according to [27]. However, a long nozzle increases the lever arm and, thus, the effect of buffeting, which is of course an additional risk. To overcome this issue, passive flow control devices at the end of the main body could be used to enhance shear layer mixing and to reduce pressure fluctuations at reattachment $[7,36]$.

The altitude or the pressure at which the transition between the modes takes place must be planned and maintained exactly to be able to deliver the calculated payload. To ensure mode transition at fixed altitude, the timing of the transition can be controlled by varying the thrust chamber pressure [13] or the fuel mixture ratio [50] or a secondary mass flow near the deflection point can be used [51]. Schneider et al. [41] suggest to use a combination of chamber mixture ratio as well as the cooling fluid mass flow rate to control the dual-bell mode transition. To avoid the additional risk due to mode transition during flight, it is also possible to perform transition before lift-off allowing for a potential payload gain of still $219 \mathrm{~kg}$, according to [45].

Another major difference to the single-bell nozzle is that with the dual-bell nozzle, the exhaust jet in sea-level mode is much thinner than the nozzle diameter. This leads to a recirculation region at the nozzle outlet and inside the second bell, which in combination with the external flow can cause additional instabilities. In [9], it was shown that with supersonic outer flow, the expansion around the nozzle's lip causes premature switching to altitude mode which is not yet stable, resulting in multiple transitions and re-transitions between both nozzle modes. Consequently, the transition to altitude mode should be completed before the sound barrier is crossed, with this kind of space launcher geometry. Verma et al. [53] observed a similar flip-flop mode during nozzle 
experiments with varying back pressure. They suggested that this scenario might be critical during transonic buffeting.

This work explores the interaction between the jet of a dual-bell nozzle with the outer flow in the base of a launcher. It is to be expected that the external flow has a significant influence on the nozzle flow. Unfortunately, so far there are only numerical simulations [3, 24, 41], experiments in still air [27, 40, 52], experiments in chambers with variable pressure [53] and experiments with supersonic external flow [2]. At this point, the present work is intended to make a contribution. The focus here is on the sea-level mode in subsonic outer flow, because in altitude mode, the dual-bell nozzle performs like a single bell nozzle with TIC or TOC contour. The sea-level mode with supersonic outer flow is less relevant because the mode transition should take place in subsonic outer flow for safety and efficiency reasons $[9,46]$. The model, the test facility and the experimental approaches are described in Sect. 2. Section 3 discusses the results of PIV and schlieren measurements and final conclusions are drawn in Sect. 4.

\section{Measurement set-up}

The measurements were performed in the trisonic wind tunnel at the Bundeswehr University in Munich (TWM). The TWM facility is a blow-down-type wind tunnel with a 300-mm-wide and 675-mm-high test section. Two adjustable throats, the Laval nozzle upstream of the test section and the diffuser further downstream, enable an operating range of Mach numbers from 0.2 to 3.0. The facility has two tanks with a total volume of $356 \mathrm{~m}^{3}$ that are pressurized with dry air up to 20 bar above ambient pressure. To control the Reynolds number, the total pressure in the test section is varied between 1.2 and 5 bar. The free-stream turbulence level based on streamwise velocity fluctuations in the TWM test section is $1.9 \%$ at a Mach number of $M=0.3$ and reduces with increasing Mach number reaching $0.45 \%$ at $M=3.0$. More details about the facility and its characterization are provided in [37, 39].

The generic 2D space launcher model consists of a 150 -mm-long nose followed by $102.5-\mathrm{mm}$-long flat main body and a $35-\mathrm{mm}$-long splitter plate with integrated nozzle, as sketched in Fig. 2. It is symmetric about its horizontal plane and spans across the entire test section width of $300 \mathrm{~mm}$. The nose design and its smooth connection to the main body ensure a shock free flow around the model up to $M=0.8$. The thickness of the forebody and the splitter plate is $25 \mathrm{~mm}$ and $15 \mathrm{~mm}$, respectively. The forebody geometry is the same as that used in $[8,35,47]$. In the center of the splitter plate, a 2D dual-bell nozzle is integrated. The nozzle has a width of $56 \mathrm{~mm}$ while the height of the throat, at the contour deflection and at the nozzle exit are $3.26 \mathrm{~mm}$,

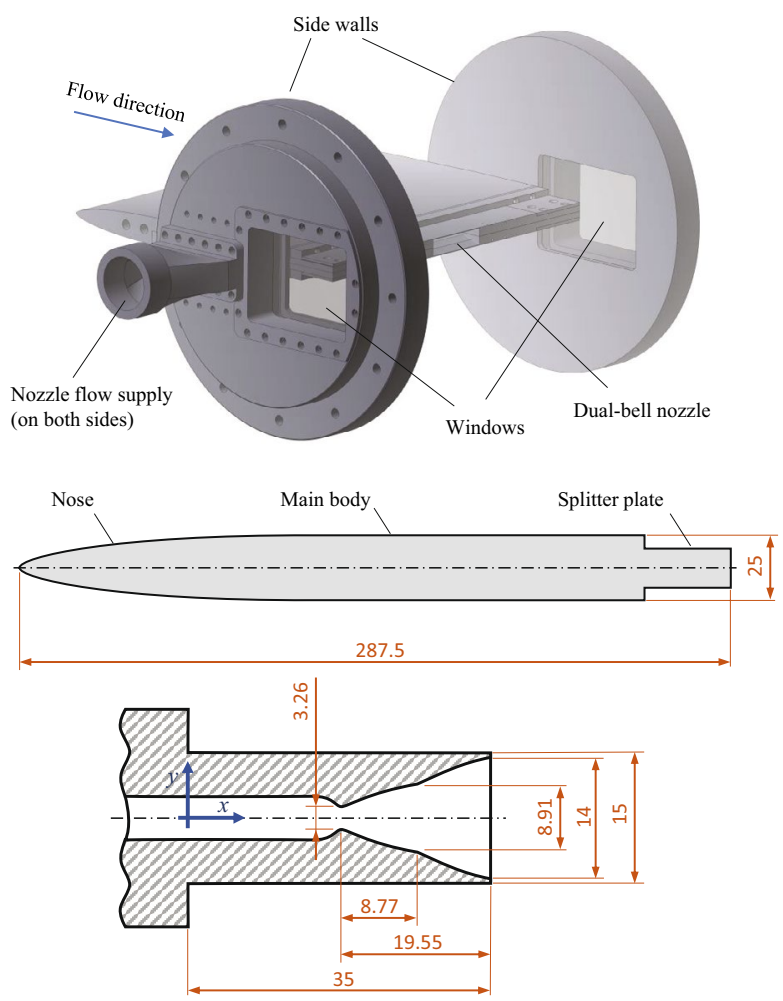

Fig. 2 Sketch of the 2D generic space launcher equipped with a dualbell nozzle. Units are given in millimeters. The geometry of the nose and the nozzle are provided in the supplementary material

$8.91 \mathrm{~mm}$ and $14.0 \mathrm{~mm}$, respectively (see Fig. 2). Thus, the expansion ratio is 4.29 leading to a design Mach number of 2.73 in altitude mode. A total temperature of $T_{0}=285 \mathrm{~K}$ leads to a design exit velocity of $u_{\mathrm{e}}=585 \mathrm{~m} / \mathrm{s}$, which is used for normalization of the results.

The two-dimensional model is ideally suited for the rectangular test section of the TWM facility. Additionally, only a 2D model allows for symmetric inflow conditions for the outer flow and the nozzle flow. Experiments with the same forebody geometry confirmed the two-dimensionality and characterized the boundary layer at the end of the main body $[6,8,35]$. Axisymmetric models are usually mounted by means of struts $[15,31,42]$ which might disturb the outer flow. However, real space launchers are based on axisymmetric geometries. Thus, it is important to keep in mind that some of the results could be caused by the planar geometry of the model.

The nozzle's thrust chamber is fed by two hoses (2-inch diameter), one on either side of the model [9]. To control the nozzle flow state, the total pressure in the nozzle settling chamber $p_{0 \text {,nozzle }}$ as well as the static pressure in the outer flow $p_{\infty}$ can be adjusted. While the first value can be varied between 1.5 bar and 10 bar the latter one depends on Mach number $M$ and total pressure $p_{0}$ of the outer flow. The nozzle pressure ratio NPR is used to characterize the nozzle flow state: 


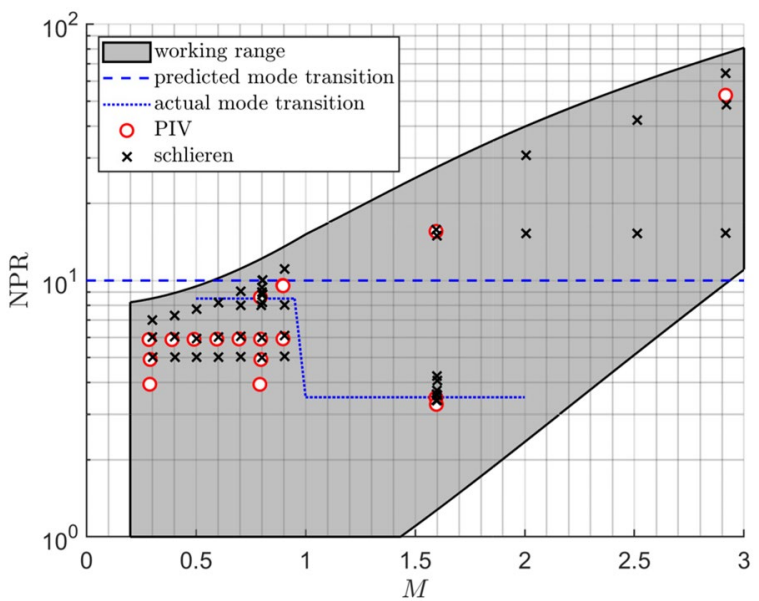

Fig. 3 Usable nozzle pressure range NPR as a function of the Mach number $M$ for the TWM facility together with the performed PIV and schlieren measurements. The dashed and the dotted line indicate the predicted and the actual transition between sea-level mode and altitude mode

$\mathrm{NPR}=\frac{p_{0, \text { nozzle }}}{p_{\infty}}=\frac{p_{0, \text { nozzle }}}{p_{0}}\left(1+\frac{\kappa-1}{2} M^{2}\right)^{\frac{\kappa}{\kappa-1}}$,

where the heat capacity ratio is $\kappa=1.4$. Figure 3 illustrates the NPR working range of the TWM model with respect to the Mach number of the outer flow. The nozzle contour was designed by Chloé Génin to perform transition from sea-level mode to altitude mode at NPR $\approx 10$ without outer flow. The design method is discussed in [14]. The contour consists of a TIC base nozzle and a positive pressure gradient nozzle extension.

To investigate the underlying flow dynamics, high repetition rate schlieren measurements of the generic space launcher's base flow were performed. Schlieren measurements visualize changes in the density gradient of the flow. Therefore, this technique is well suited to detect shear layers as well as shocks and expansions. The TWM test section is illuminated with light from a high-power LED ( $\lambda=462 \mathrm{~nm}$, CBT-120-B-C11-KM301 by Luminus Devices Inc.) focused onto a 2-mm-wide vertical slit located in the focal point of a spherical concave mirror with a focal length of $4000 \mathrm{~mm}$ which results in an illumination with a high degree of parallelism. The diffraction due to density changes inside the flow is analyzed by means of a knife edge located in the focal plane of a second mirror on the other side of the test section. For all the discussed measurements, the knife edge is oriented in vertical direction to analyze the changes of density in streamwise direction. Without the flow turned on, the knife edge position was set to let through $75 \%$ of the light intensity to avoid too dark regions in the schlieren images by reducing the sensitivity, according to [44].
The filtered light was imaged onto the sensor of a highspeed camera (Phantom V2640, by Vision Research Inc.), which was operated at a recording rate of $40 \mathrm{kHz}$ with an image size of $1024 \times 576$ pixel corresponding to $85 \times 48$ $\mathrm{mm}^{2}$. Aliasing due to contribution of higher frequencies was minimized by setting the exposure time to its maximum, which is $24 \mu \mathrm{s}$. To avoid too bright regions in the schlieren images, the LED power was adjusted such that the intensity on the sensor reached $30 \%$ of the maximum camera range with the flow turned off. More details about the TWM schlieren system can be found in $[17,38]$.

In contrast to schlieren measurement, particle image velocimetry (PIV) provides results in a plane rather than averaged over a volume. Additionally, the flow velocity allows for the determination of statistical values that are useful for comparison with other experiments or flow simulations. For the PIV measurements the flow was seeded with Di-Ethyl-Hexyl-Sebacat (DEHS) tracer particles with a mean diameter of $1 \mu \mathrm{m}$, as described by [21]. The size of the particles is a compromise between visibility and particle response time [29]. The particles in the TWM test section were illuminated from the top with a light sheet generated by a PIV double pulse laser (DM 150-532, by Photonics Industries Inc.) and the scattered light was recorded by means of a high-speed camera (Phantom V2640, by Vision Research Inc.) equipped with a 100-mm lens (Planar T2,0/100 ZF-I macro, by Zeiss) and a $2 \times$ tele-converter (DGX $2 \times$, by Kenko). Double images with a size of $2048 \times 1240$ pixel (corresponding to $72 \times 44 \mathrm{~mm}^{2}$ ) were recorded with $5 \mathrm{kHz}$.

To analyze the effect of nozzle pressure ratio on the flow dynamics and interactions between the nozzle flow and the base flow, schlieren measurements as well as PIV measurements were performed over a large range of NPR and $M$. The markers in Fig. 3 summarize the different wind tunnel runs. For each measurement condition, 10,000 Schlieren images or 10,000 PIV double images were recorded and evaluated.

\section{Results and Discussions}

\subsection{Schlieren visualization}

Thanks to the simple setup and the easy to handle data, schlieren measurements are a powerful tool for the analysis of compressible flows. However, the results suffer from two drawbacks. First, the intensity of schlieren images provides only qualitative results, if not calibrated [19]. Second, the results are averaged in the direction of light propagation over the measurement volume. Nevertheless, the location of shocks, expansions and strong shear layers can be reliably determined. Since 10,000 schlieren images were recorded at $40 \mathrm{kHz}$ for each case, the motion over time and statistics of these features can also be determined. Figure 4 illustrates 

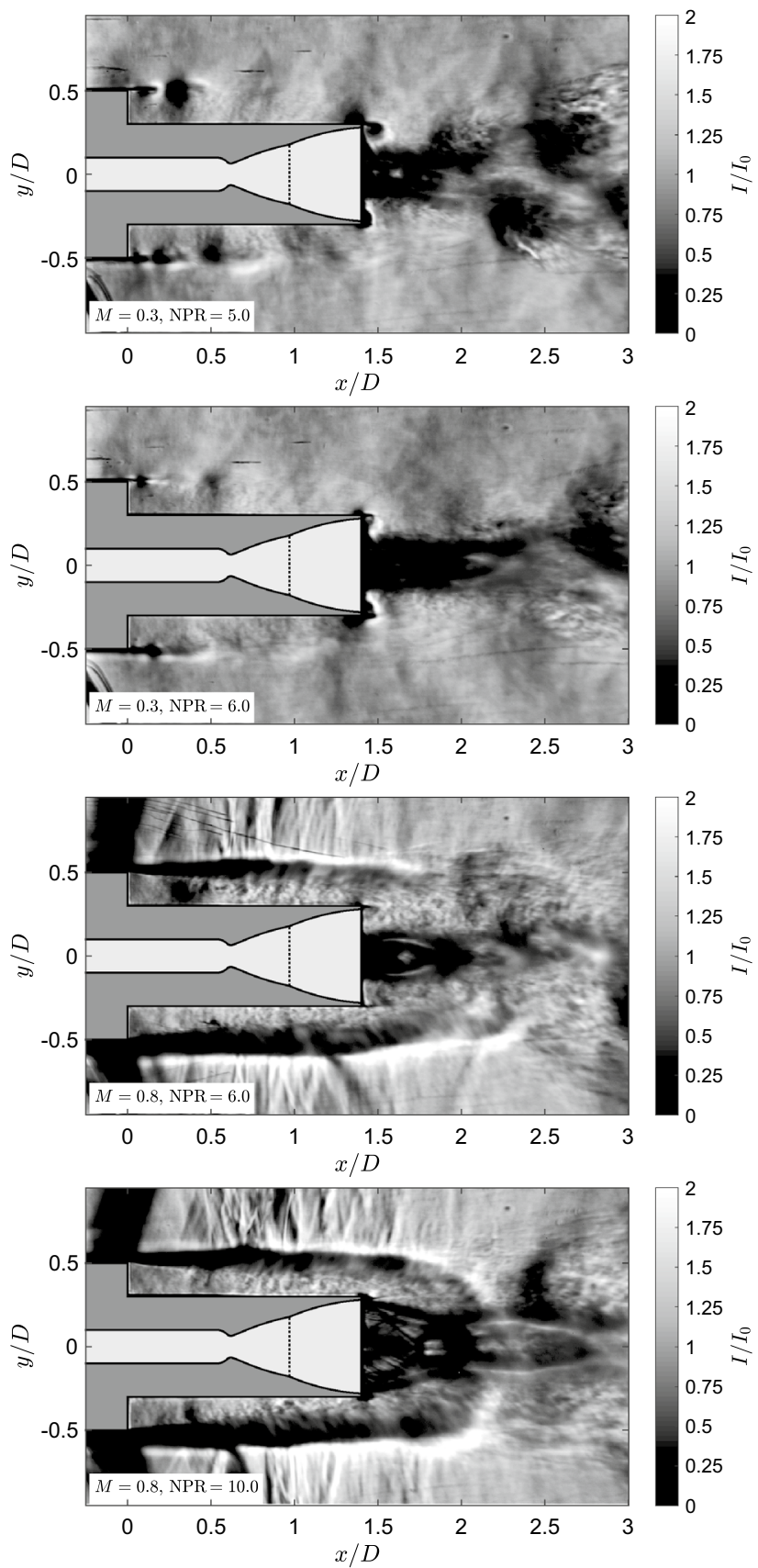
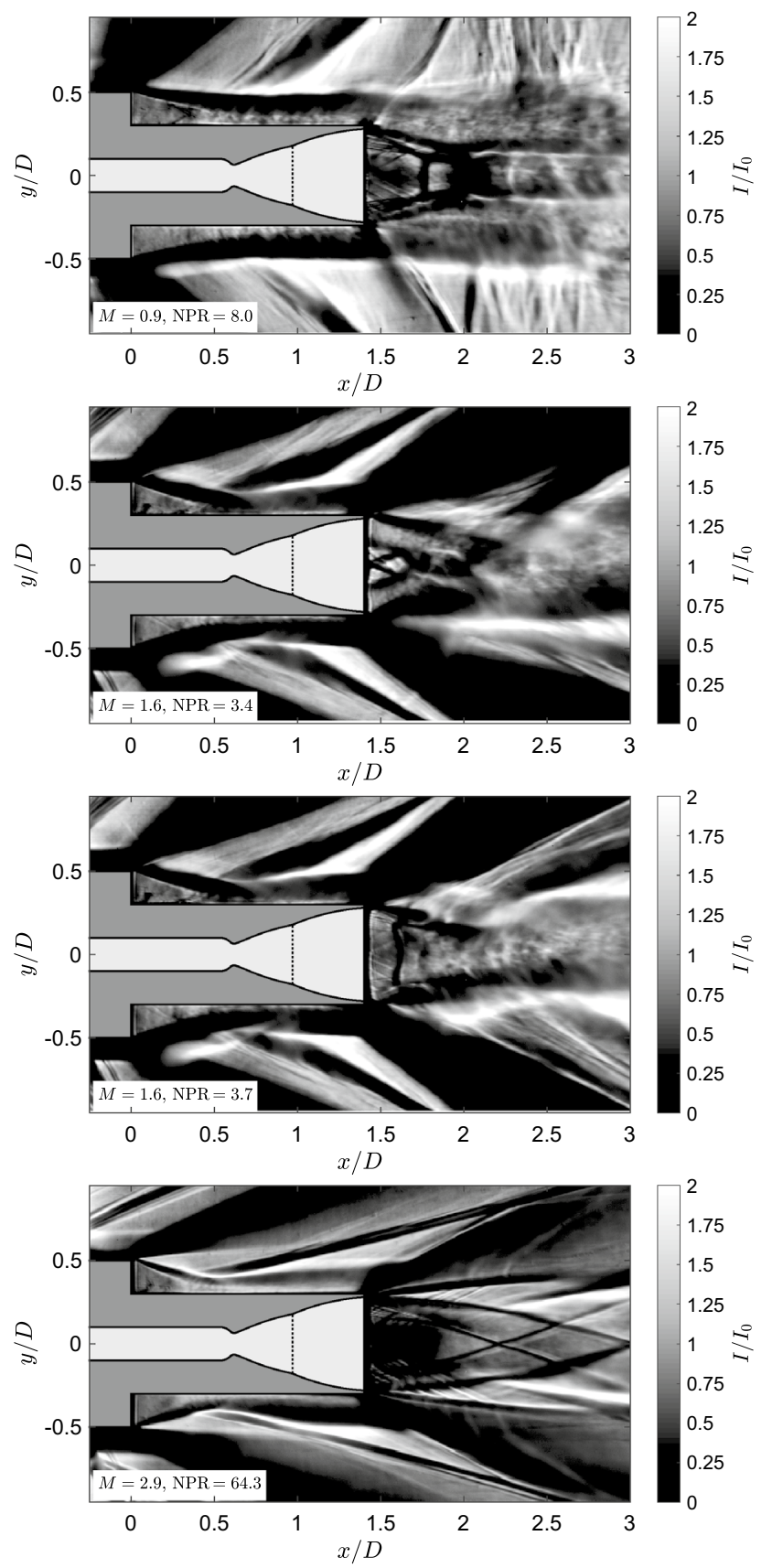

Fig. 4 Examples of instantaneous schlieren images recorded at different nozzle pressure ratios NPR and Mach numbers $M$. Supplementary videos are provided online

instantaneous schlieren images at different Mach numbers and nozzle pressure ratios. Comparing the different Mach number cases, only at $M=0.3$ individual vortices generated in the separated shear layer at the end of the main body are visible. This indicates that these structures are highly homogeneous in spanwise direction at $M=0.3$. For larger Mach numbers the exposure time of $24 \mu$ s is too long to identify individual vortices and/or the homogeneity in spanwise direction no longer exists.

Regarding the nozzle flow, Fig. 4 provides an overview of the different states. For $M=0.3$ and 0.5, the dual-bell nozzle is operated in sea-level mode, for $M=0,9$ and 2.9 it runs in altitude mode and for $M=0.8$ and 1.6 both modes are shown in the figure. Additionally, it can be seen that the various NPR values lead to different behavior at 

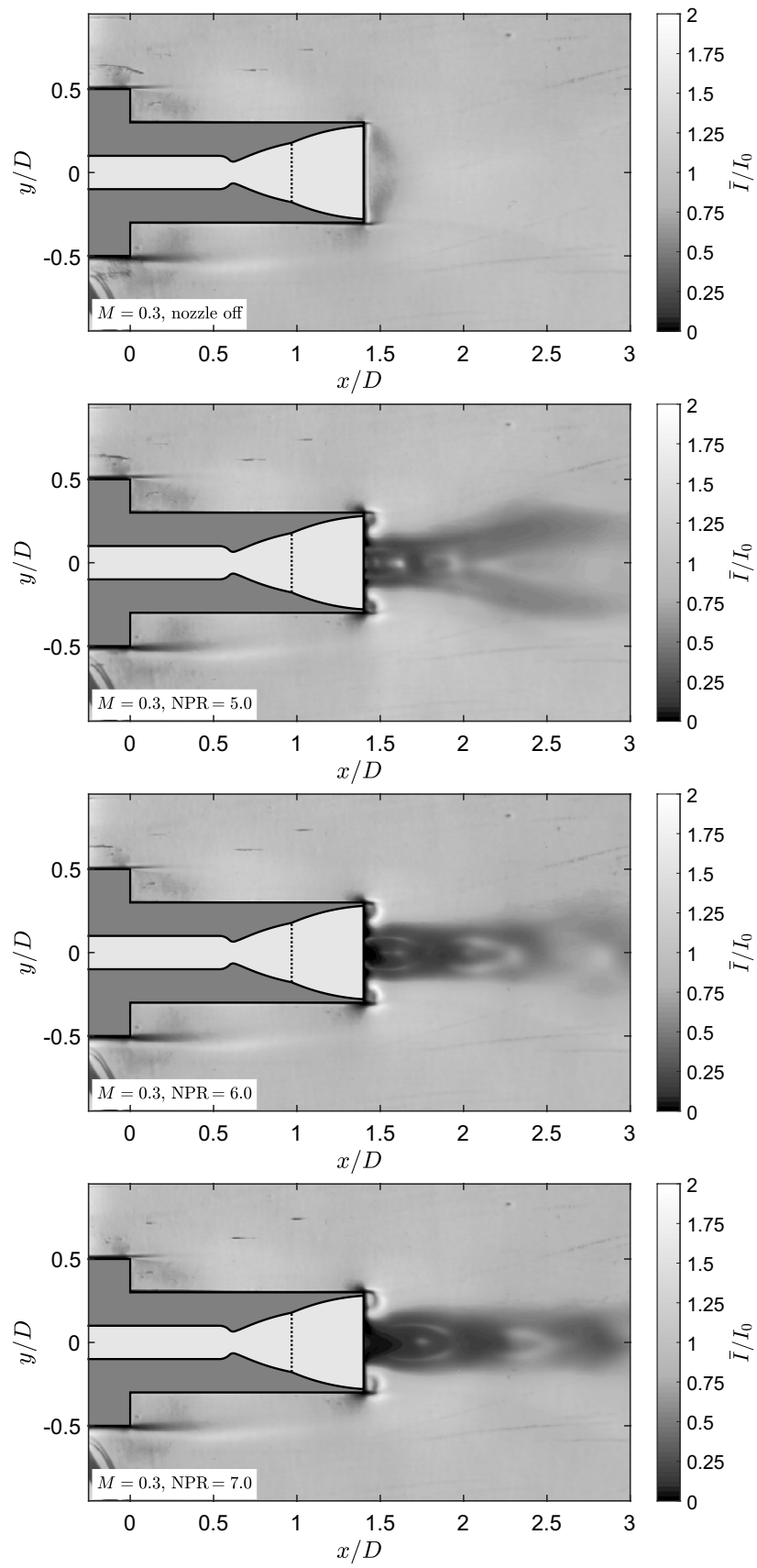
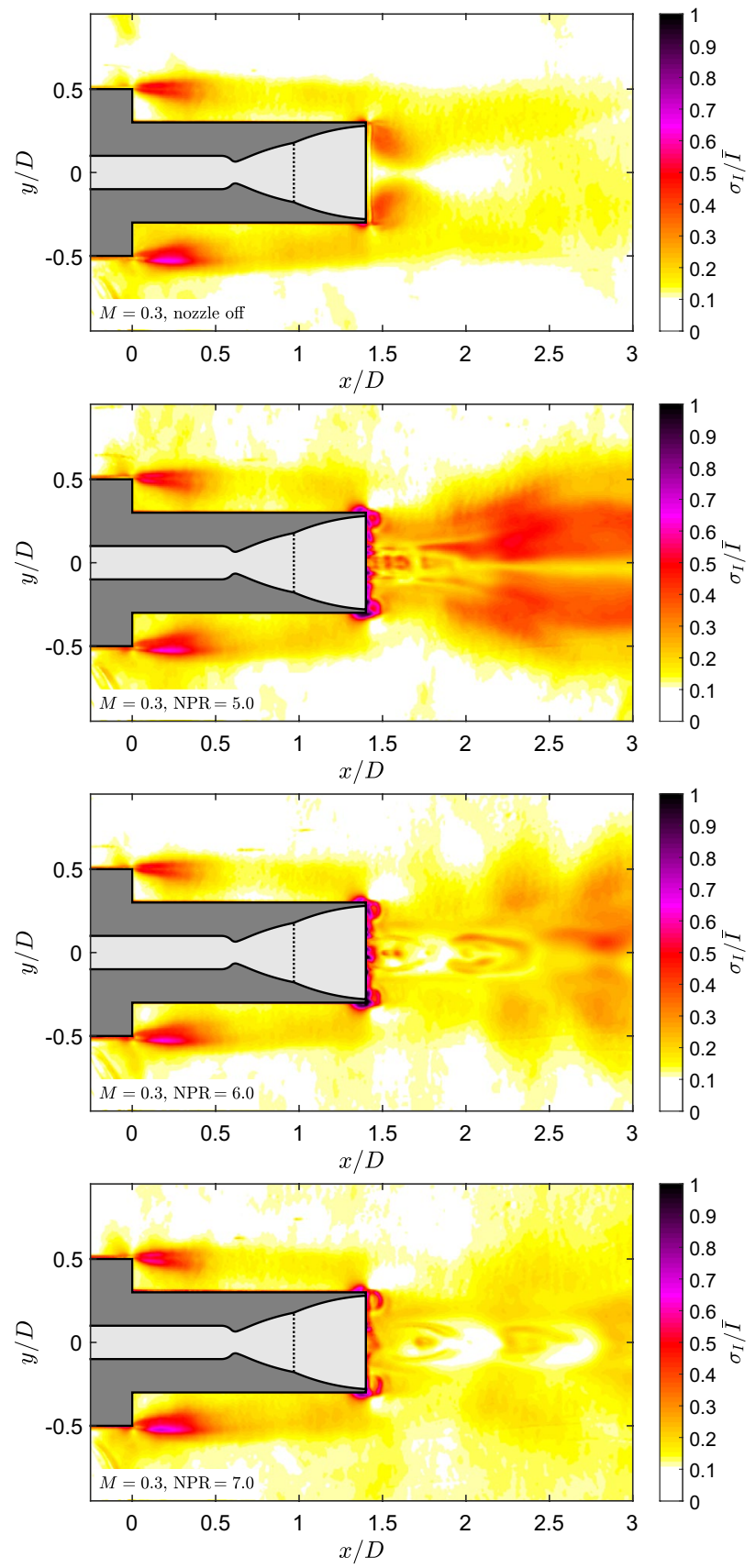

Fig. 5 Averaged schlieren intensity (left) and corresponding standard deviation (right) for $M=0.3$ and different values of NPR

the investigated Mach numbers. This will be discussed in detail in the following sections.

\subsubsection{Schlieren statistics}

With the aim to determine the mean flow topology and to identify regions and cases with strong density fluctuations, the mean schlieren intensity as well as its standard deviation was computed. The averaged schlieren images are used to determine the nozzle flow state as well as expansions and shocks in the supersonic outer flow. The schlieren intensity standard deviations provide information about the oscillation of the detached shear layer of the step as well as of the exhaust jet. Figures 5, 6 and 7 show the results for $M=0.3$, 0.8 and 1.6, respectively. For these Mach numbers, the case without nozzle flow is shown as reference together with different NPR values.

For the $M=0.3$ case, illustrated in Fig. 5, high-intensity fluctuations are present in the separating shear layers emerging from the end of the main body $(x / d=0)$ and at 

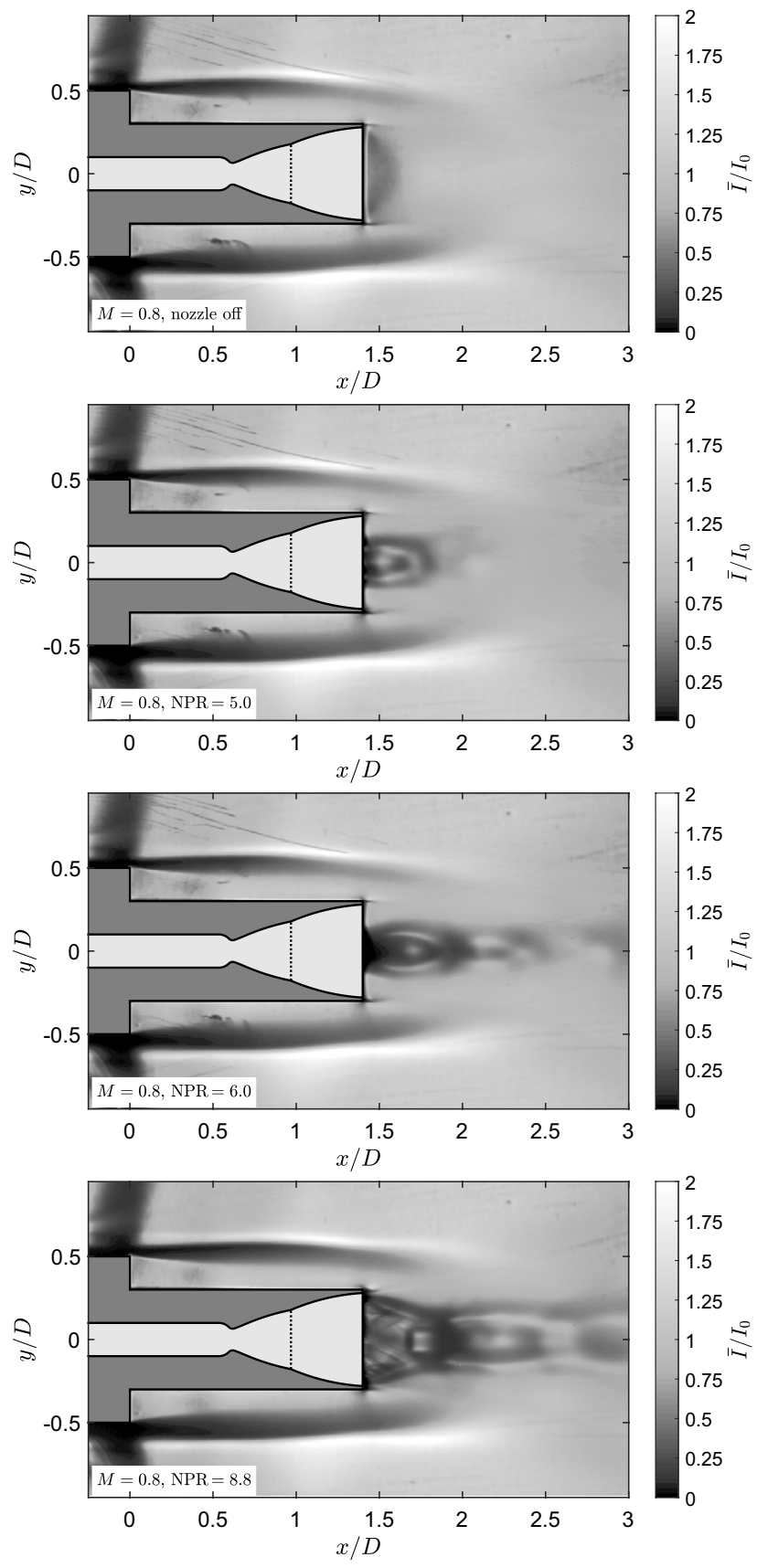
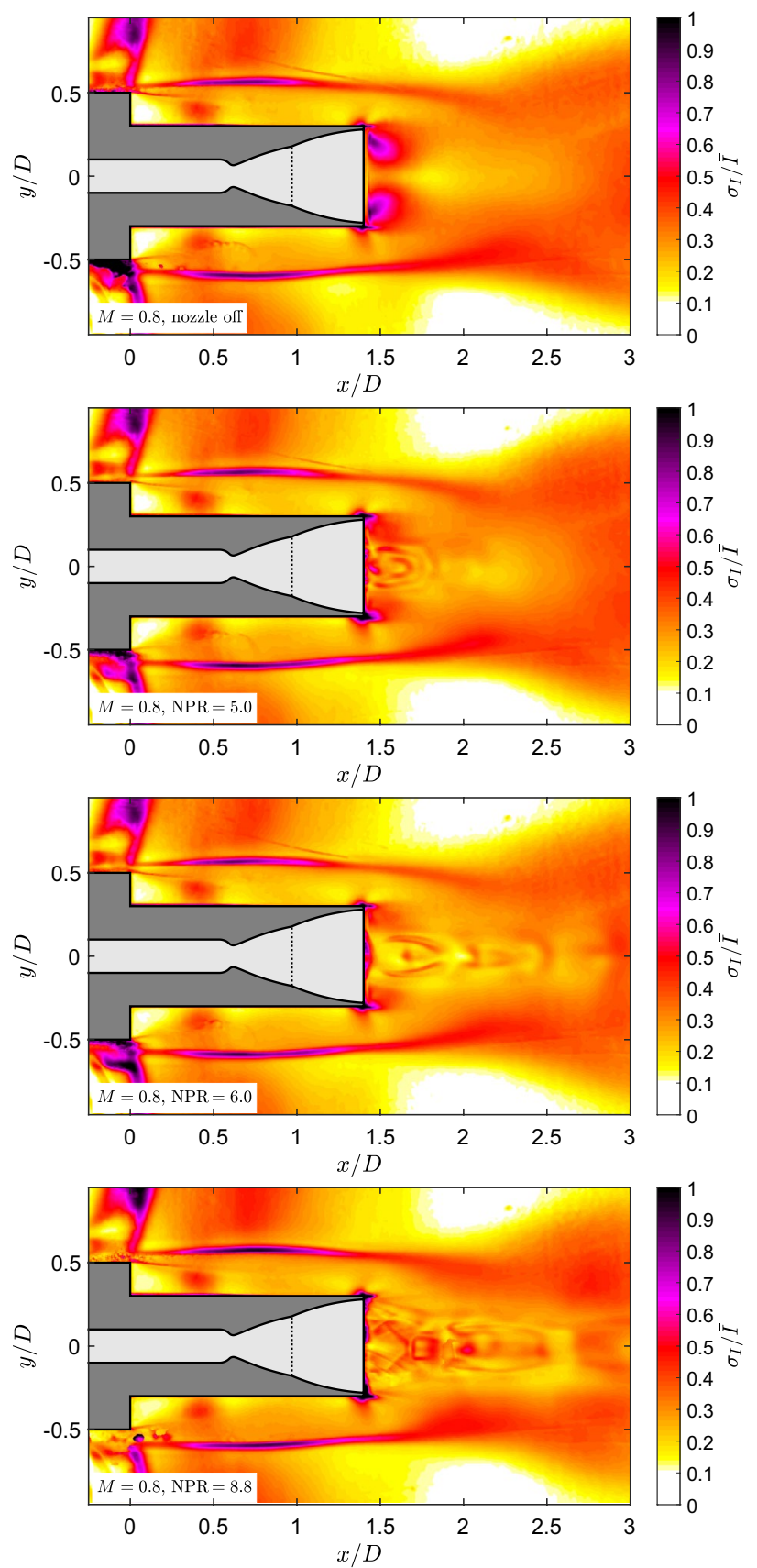

Fig. 6 Averaged schlieren intensity (left) and corresponding standard deviation (right) for $M=0.8$ and different values of NPR

the nozzle's lip $(x / d=1.4)$. The outer flow is rather independent of NPR, while the jet itself undergoes significant changes. For all three tested NPR values shown in Fig. 5, the dual-bell nozzle is operated in sea-level mode. Thus, the jet flow separates at the contour deflection and a recirculation region is formed in the second bell and outside of it (see Fig. 1). For NPR= 5.0 the nozzle flow shows a strong spread accompanied by large fluctuations. This is caused by an oscillation of the jet, which is associated with the formation of large vortices on both sides, refer to the top left of Fig. 4. Increasing the nozzle pressure ratio to 6.0 (second row from bottom in Fig. 5) reduces the fluctuations significantly. However, the spatial distribution of the intensity standard deviation shows now a periodic pattern with local minima and maxima in streamwise direction. This form indicates that the nozzle flow performs so-called jet screech [30]. The jet performs strong oscillations, with the middle section of each shock cell oscillating less than the ends. This can be clearly seen in the supplementary videos provided with Fig. 4. This motion is linked to a 

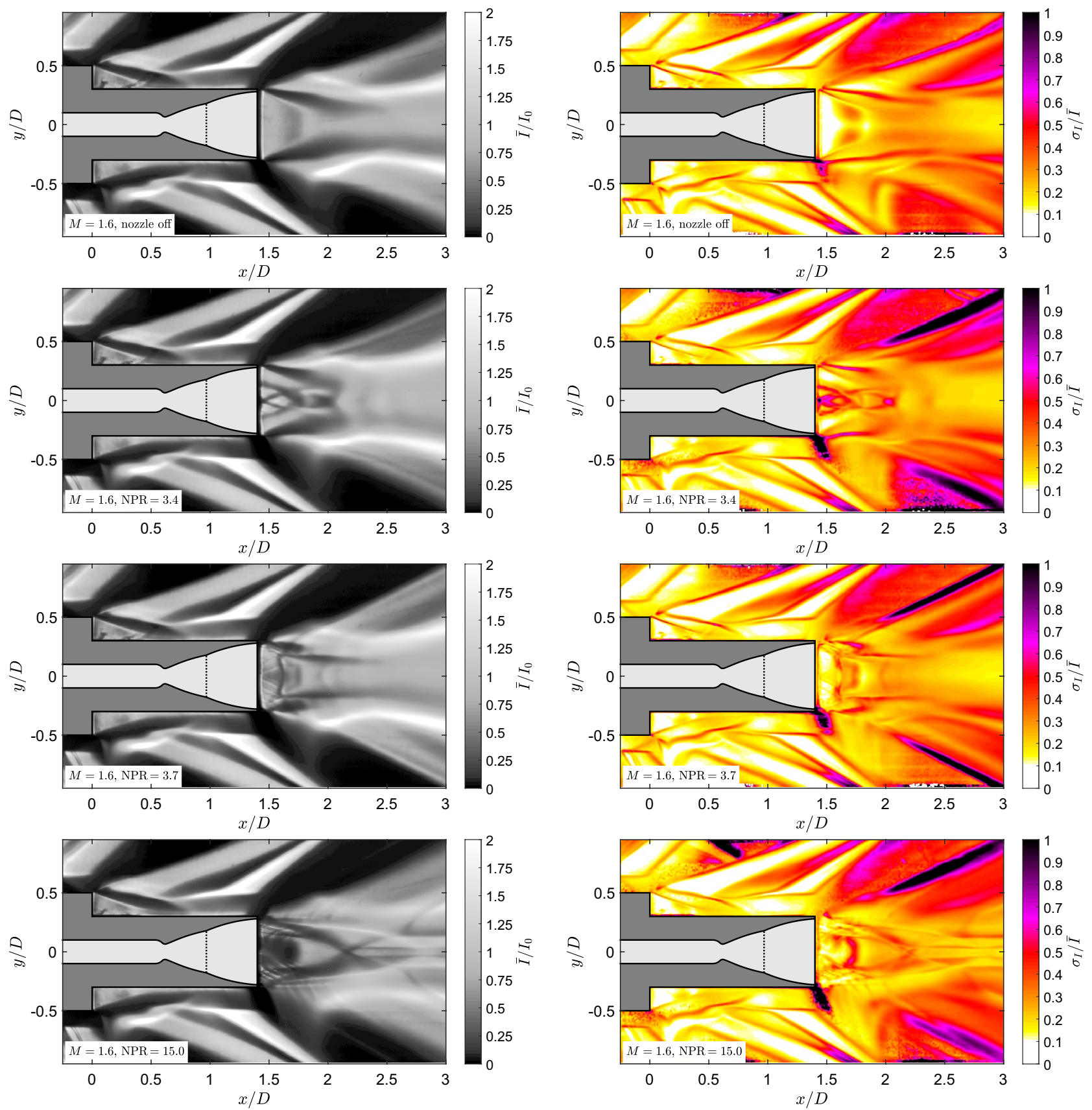

Fig. 7 Averaged schlieren intensity (left) and corresponding standard deviation (right) for $M=1.6$ and different values of NPR

certain frequency, as will be discussed in the following subsection. For NPR=7.0 (bottom row in Fig. 5) the pattern is no longer visible.

For $M=0.8$, the schlieren signal of the shear layers emerging from the end of the main body is much stronger and its fluctuation is clearly visible even well behind the model, see top row in Fig. 6. With the nozzle flow turned on, the region with high standard deviation remains unchanged because the signal is integrated across the entire spanwise extent of the test section $(300 \mathrm{~mm})$ while the nozzle width is only $56 \mathrm{~mm}$. Regarding the jet, it can be seen from the figure that at NPR= 5.0 and 6.0, the nozzle is operated in sea-level mode while it is in altitude mode for $\mathrm{NPR}=8.8$. Without outer flow, the altitude mode was only reached for NPR $\geq 10$. It is important to note that the mode transition NPR is shifted towards smaller values with the outer flow turned on. This change in mode transition NPR is caused by the aspiration drag [20] which causes a reduction of the static pressure near the nozzle exit relative to the freestream, as shown in [9]. The standard deviation of the schlieren intensity in Fig. 6 

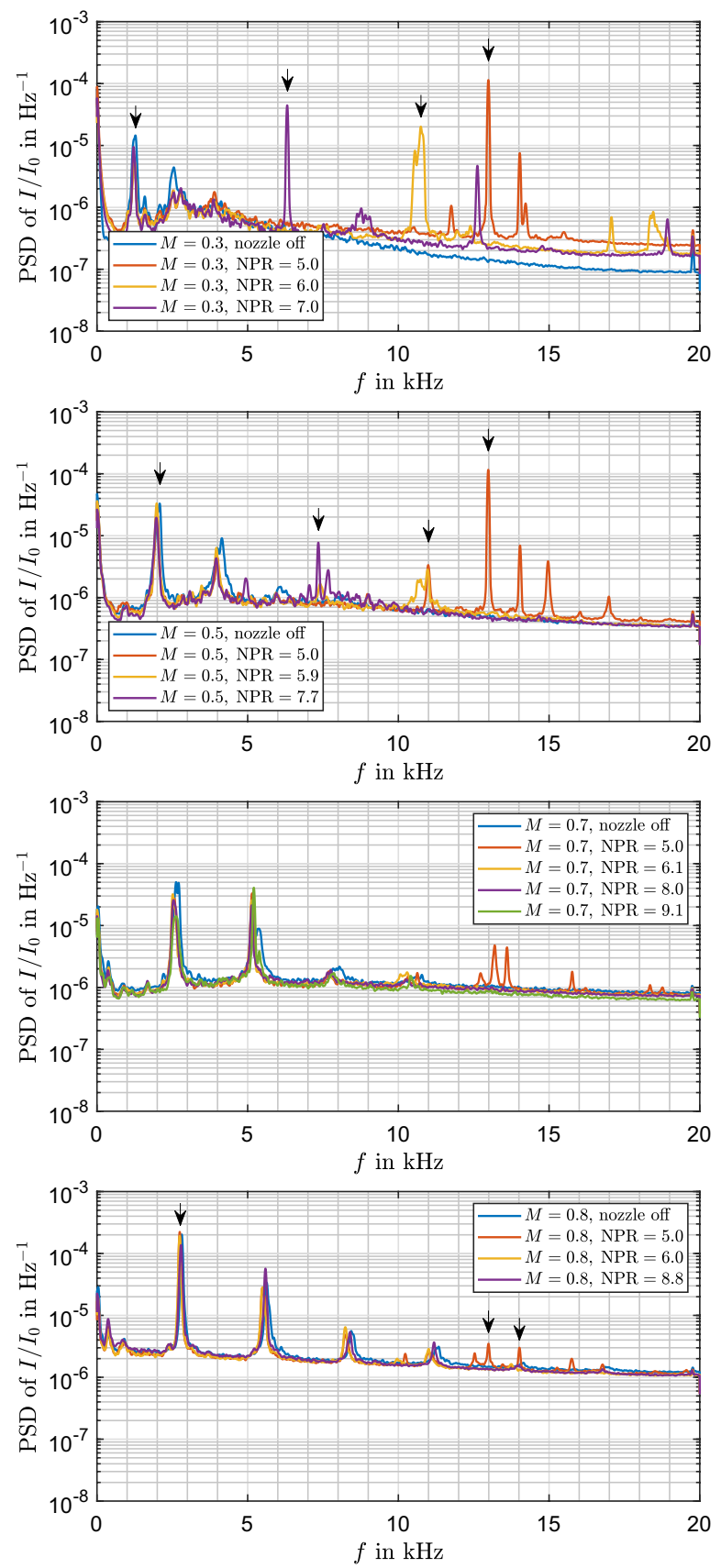
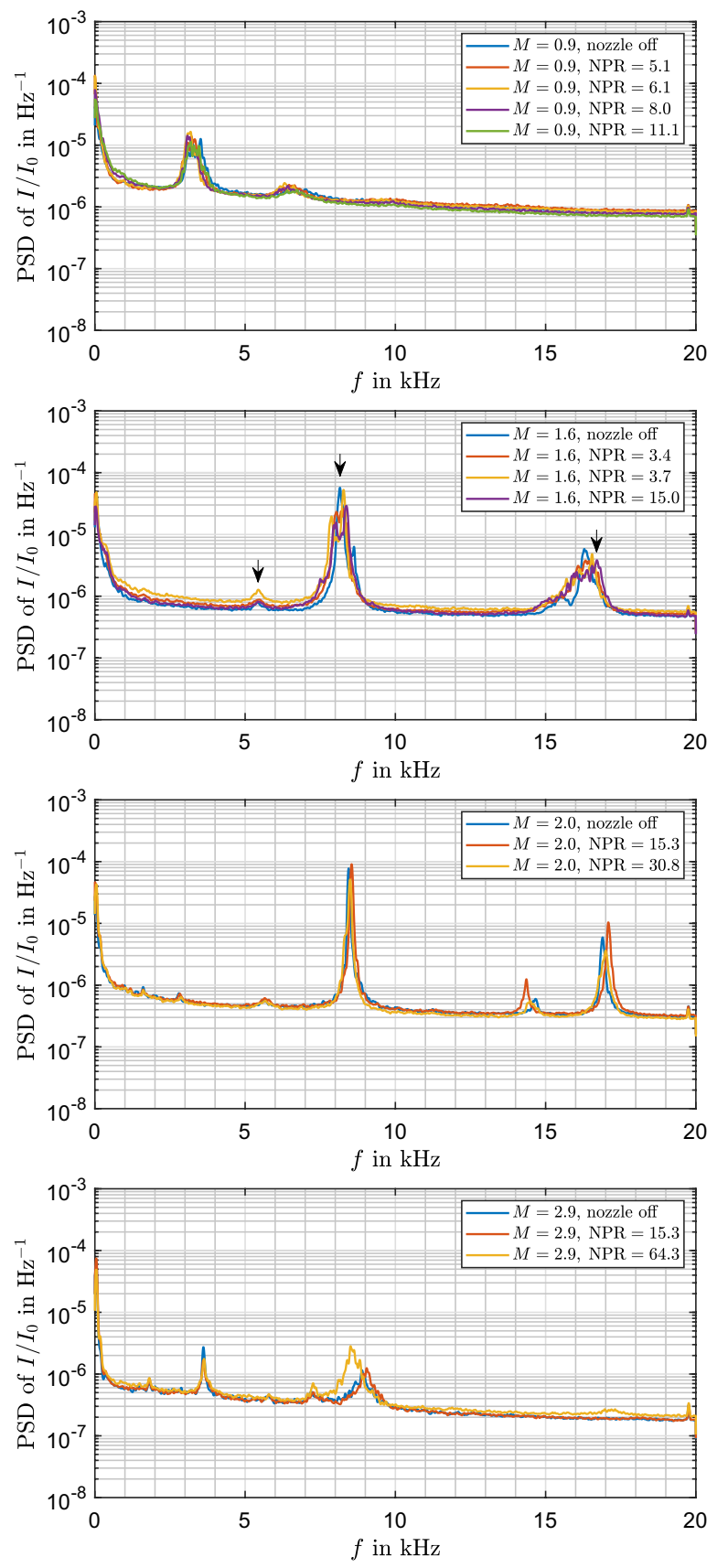

Fig. 8 Spatial averaged power spectral density of the schlieren intensity for different Mach numbers $M$ and nozzle pressure ratios NPR. The black arrows indicate the peaks for which the spatial distribution is discussed in the following

does not show any fixed pattern like for the $M=0.3$ case. Whether or not the nozzle performs screeching cannot be determined from the figure. The spectral analysis will contribute to the clarification of this point in the next subsection.

For $M=1.6$, the spatial distribution of the averaged schlieren signal and its standard deviation changes, as expected for supersonic flow. It can be seen in Fig. 7, that high fluctuations of the schlieren intensity appear at the edges of homogeneous regions, indicating oscillation of shocks and expansion regions. As before, the outer flow is rather independent of the jet flow. The jet is operated in altitude mode already at NPR $=3.7$. The reason for the nozzle mode transition at much lower NPR values compared to subsonic condition is the massively reduced static pressure due to flow expansion around the nozzle's lip [9]. 


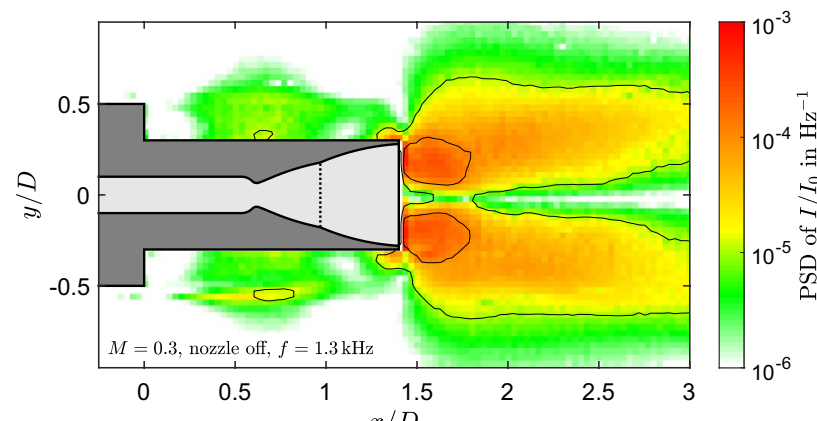

$x / D$

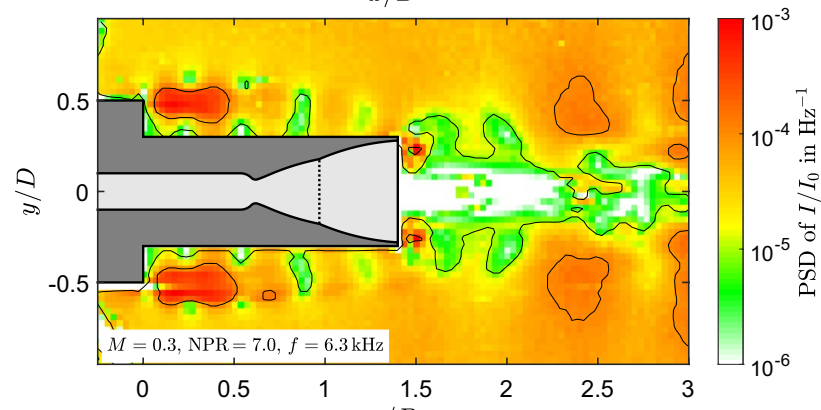

$x / D$
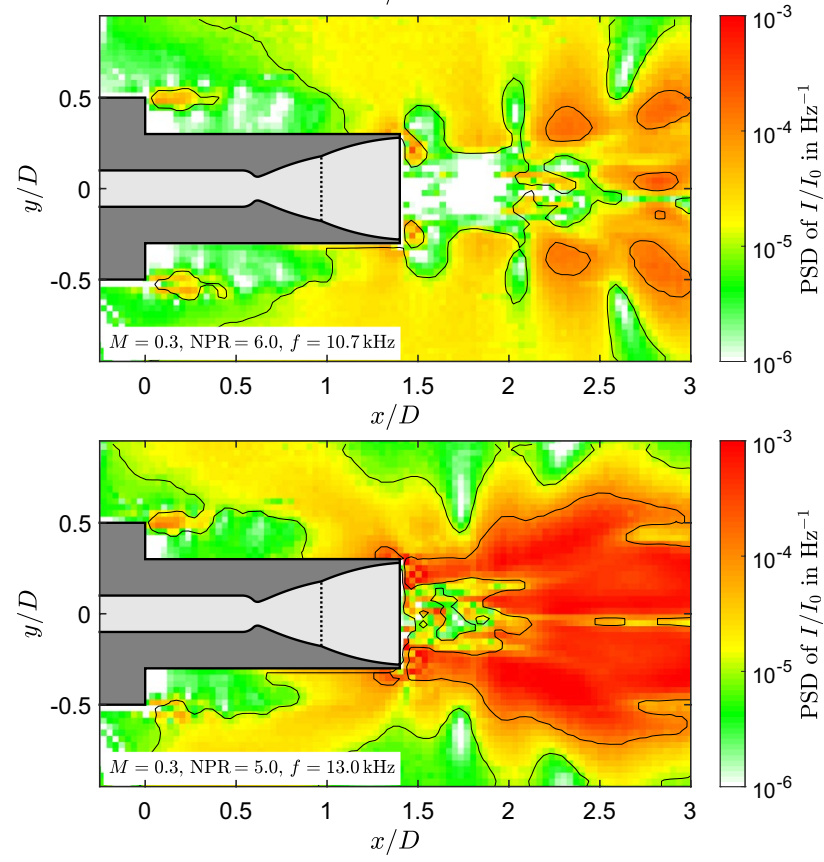

Fig. 9 Spatial distribution of the schlieren signal spectral content for fixed frequencies at $M=0.3$

\subsubsection{Spectral analysis}

To further investigate the underlying phenomena that cause the high fluctuations in the schlieren signal, the power spectral density PSD was computed from the data sets using the method of [57]. The aim of the spectral analysis is to identify periodic phenomena as well as their corresponding frequencies and spatial organization. A band-pass filtering based on the dominant frequencies allows for comparing spatial modes excited at different frequencies for different nozzle pressure ratios and Mach numbers. Figure 8 shows the spatially averaged PSD for subsonic and supersonic Mach numbers. Besides different NPR values, the case without nozzle flow is always included as reference. The figure clearly shows that the nozzle pressure ratio has strong impact on the flow dynamics for the low subsonic Mach numbers; while for $M>0.7$, only minor changes are observed between the different NPR values.

To see which regions contribute to the peaks in the PSD, Figs. 9-12 show the spatial distribution of some of the most pronounced frequencies, marked with arrows in Fig. 8. For $M=0.3$, the peak around $1.3 \mathrm{kHz}$ occurs both with the nozzle flow switched on and off. Consequently, this is not an effect of the nozzle flow. The spatial distribution of the spectral intensity suggests that this frequency is related to the vortex shedding from the end of the main body. Theses eddies interact with the nozzle fairing and generate even stronger contributing eddies downstream of the nozzle dummy. Since the nozzle exit spans only $56 \mathrm{~mm}$ out of the $300-\mathrm{mm}$ spanwise width of the test section, this mode is also visible with the jet turned on for all NPR values.

The other three frequencies shown for $M=0.3$ in Fig. 9 only appear for a certain NPR. Consequently, these modes are generated by the nozzle flow. It can be seen from the figure that local mimima and maxima appear for the schlieren intensity fluctuations in the streamwise direction. For $\mathrm{NPR}=5.0$ and 6.0 (bottom two rows in Fig. 9), the strongest contributions are generated downstream of the nozzle exit, while for NPR=7.0 (second rows in Fig. 9) a region in the early shear layer emerging from the main body shows the strongest contribution. Thus, for $M=0.3$ and NPR=7.0, the jet strongly interacts with the space launcher base flow. The periodic patterns in the PSD distributions with local minima and maxima for all NPR values indicate distinctive jet screech.

The spatial contribution to the PSD for $M=0.5$ in Fig. 10 is similar to the $M=0.3$ cases. There is a common frequency generated by the main flow at $2.1 \mathrm{kHz}$ as well as individual peaks for the tested NPR values. For NPR $=7.7$ a region in the early shear layer emerging from the main body shows the strongest contribution, similar to the NPR $=7.0$ case for $M=0.3$ but with reduced intensity downstream of the nozzle exit. It is interesting to note, that for $\mathrm{NPR}=5.0$, the frequency of the strongest peak at $13.0 \mathrm{kHz}$ does not change than increasing the Mach number from 0.3 to 0.5 ; while, the frequency of the other peaks increases with the Mach number.

For $M=0.8$, there is again a peak for all NPR cases at a fixed frequency, which is now shifted $2.8 \mathrm{kHz}$. It can be seen from the comparison of the top rows of Figs. 9, 10 and 11 , that the spatial distribution of the contribution to the first spectral peak is similar for all subsonic cases. As discussed 


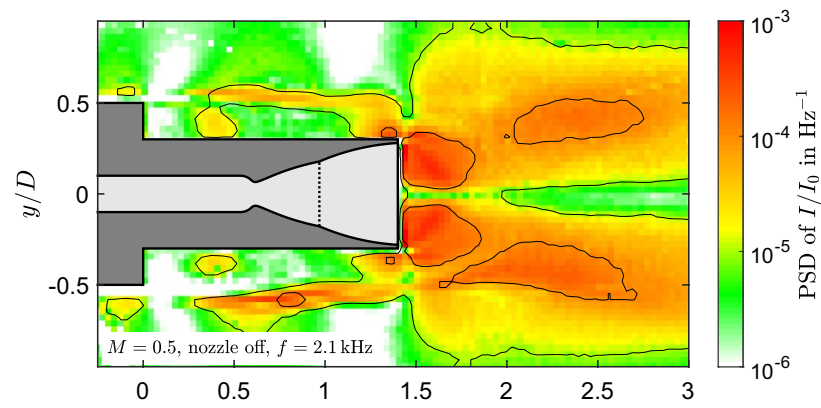

$x / D$

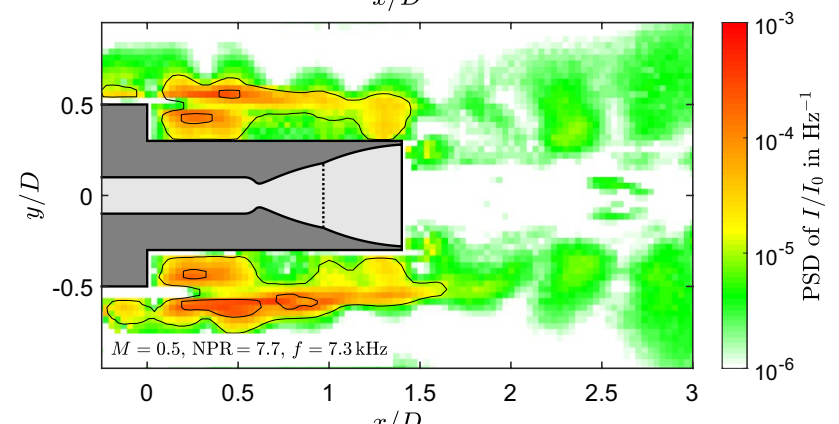

$x / D$

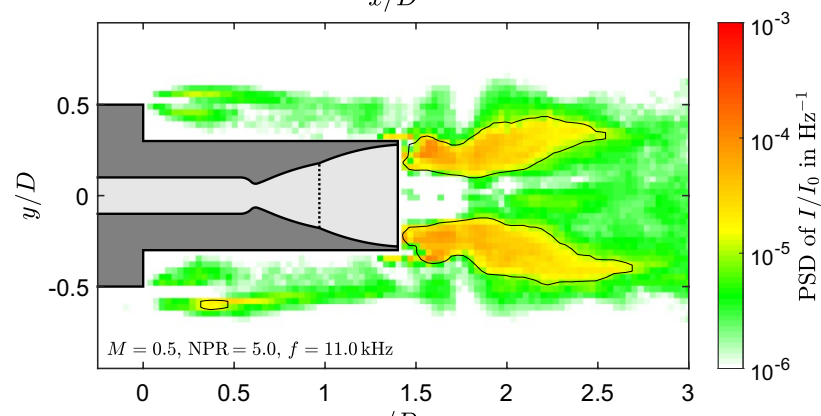

$x / D$

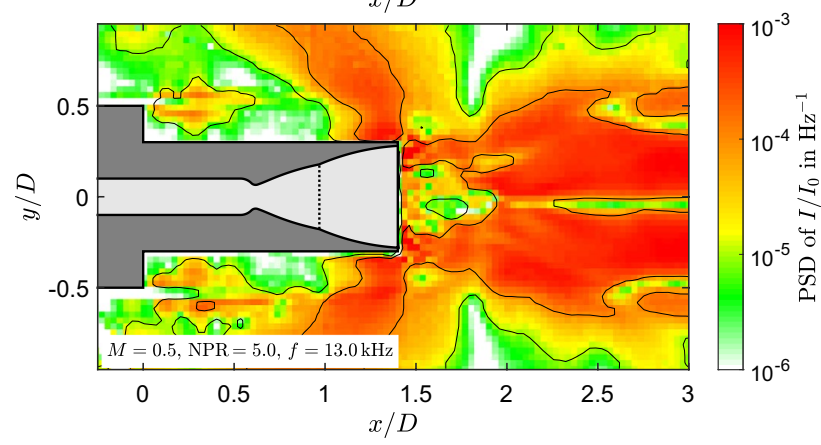

Fig. 10 Spatial distribution of the schlieren signal spectral content for fixed frequencies at $M=0.5$

earlier, this mode is related to the vortex shedding from the end of the main body. The frequnecy of this mode is increasing with the flow velocity, as expected. But the reduced frequency, based on the free-stream velocity and the model thickness, drops slightly with increasing Mach number, as shown in Fig. 13. Apart from this, there is a small contribution only for $\mathrm{NPR}=5$ at $M=0.8$, which does not also occur without nozzle flow. The corresponding frequency is again
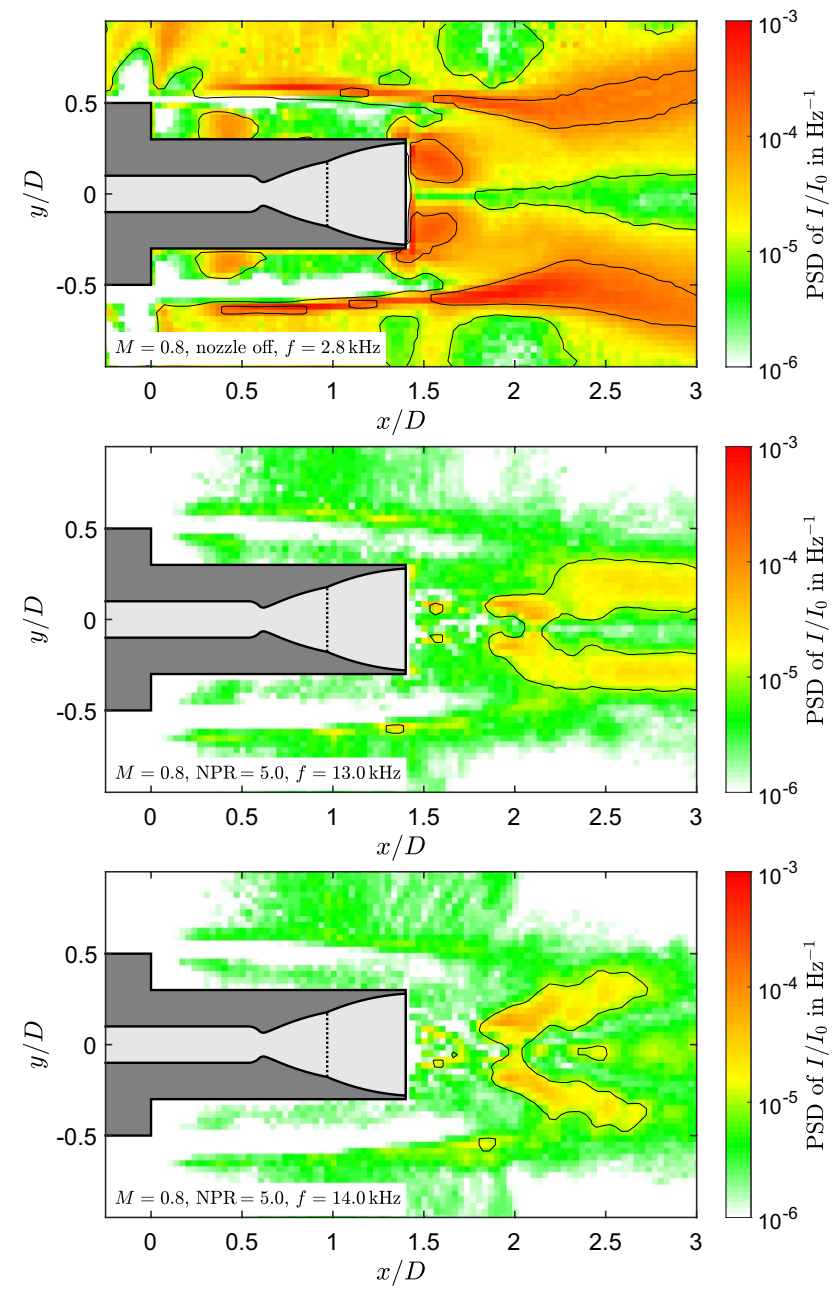

Fig. 11 Spatial distribution of the schlieren signal spectral content for fixed frequencies at $M=0.8$

13.0 $\mathrm{kHz}$ but the spatial distribution of the PSD at said frequency is significantly reduced compared to the lower Mach number cases. Only a region downstream of the first shock cell near the jet is contributing to this peak and to another peak at $14.0 \mathrm{kHz}$, shown in the bottom row of Fig. 11.

For $M=0.9$ to 2.9 (right column in Fig. 8), no significant change can be seen in the spectra between the cases with and without jet, suggesting that they are coming from the main flow. The spatial distribution from the three peaks in the spectra of the $M=1.6$ case are illustrated in Fig. 12 . It can be seen that the contributions come mainly from the region downstream of the nozzle exit. Thus, it can be concluded that with supersonic outer flow the interaction between the separated shear layer and the nozzle flow is significantly reduced. 


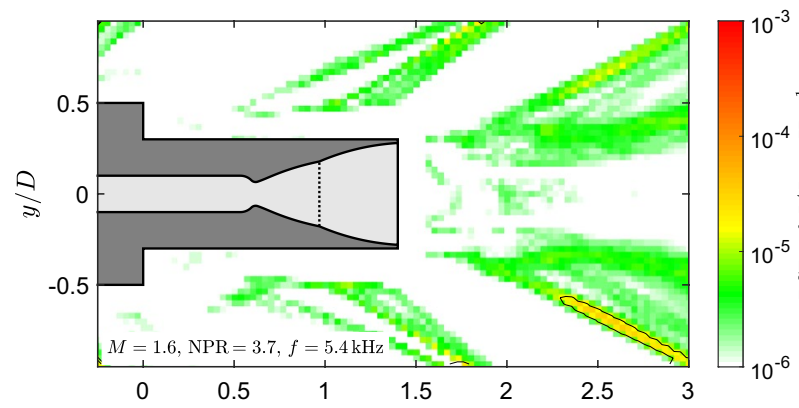

$x / D$

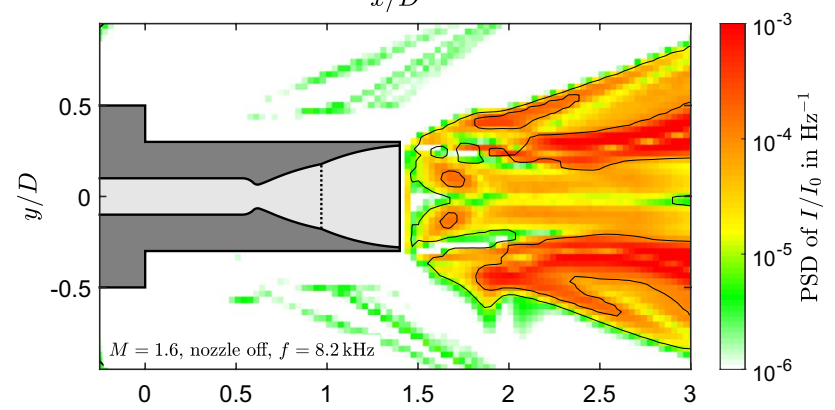

$x / D$

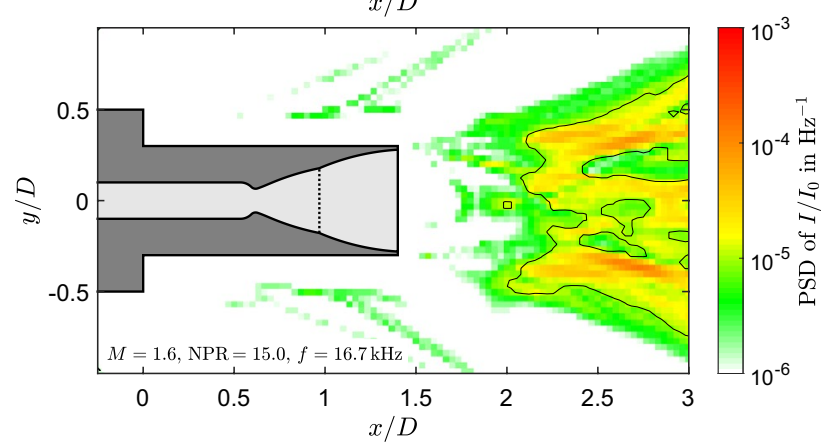

Fig. 12 Spatial distribution of the schlieren signal spectral content for fixed frequencies at $M=1.6$

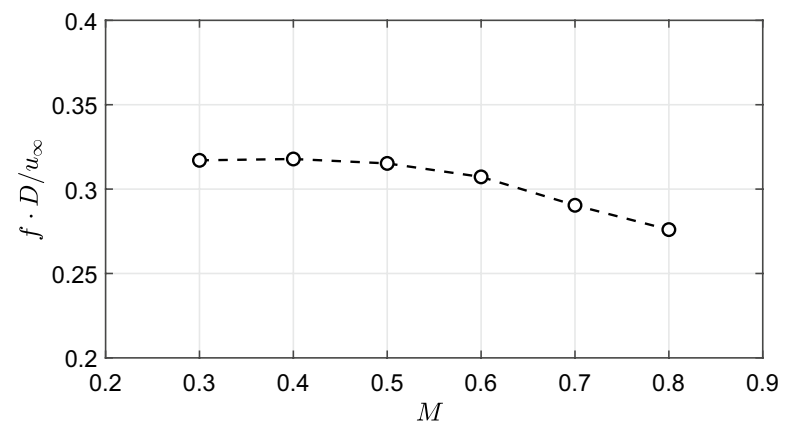

Fig. 13 Reduced frequency of the first dominant mode of the PSD from the subsonic cases in Fig. 8

\subsection{Velocity measurements}

To determine the topology of the space launcher's afterbody velocity field, PIV measurements on the center plane were performed and are discussed in this section. As before,
Table 1 Wind tunnel free-stream flow conditions for the PIV experiments. The range for total pressure $p_{0}$ and total temperature $T_{0}$ refers to the variation within the run time and between individual wind tunnel runs

\begin{tabular}{llll}
\hline $\mathrm{M}$ & $p_{0}$ in bar & $T_{0}$ in $\mathrm{K}$ & $R e_{\mathrm{D}}$ \\
\hline 0.3 & $1.3 \pm 0.005$ & $295 \pm 2$ & $2.04 \times 10^{5}$ \\
0.4 & $1.3 \pm 0.005$ & $291 \pm 2$ & $2.72 \times 10^{5}$ \\
0.5 & $1.3 \pm 0.005$ & $289 \pm 2$ & $3.32 \times 10^{5}$ \\
0.6 & $1.3 \pm 0.005$ & $289 \pm 2$ & $3.82 \times 10^{5}$ \\
0.7 & $1.3 \pm 0.005$ & $290 \pm 2$ & $4.24 \times 10^{5}$ \\
0.8 & $1.3 \pm 0.005$ & $288 \pm 2$ & $4.58 \times 10^{5}$ \\
0.9 & $1.3 \pm 0.005$ & $289 \pm 2$ & $4.85 \times 10^{5}$ \\
1.6 & $2.0 \pm 0.005$ & $288 \pm 2$ & $7.56 \times 10^{5}$ \\
\hline
\end{tabular}

the outer flow Mach number and the nozzle pressure ratio were varied with the aim to understand the interaction between nozzle flow and outer flow in more detail. Unlike the schlieren measurements, the PIV velocity fields are averaged over only $\approx 1 \mathrm{~mm}$ in spanwise direction. This is important for the characterization of the interaction, because of the limited width of the nozzle. The velocity measurements are used to determine the mean reattachment length of the separated shear layer and its dependence on the nozzle flow. Furthermore, the velocity fluctuations provide quantitative information about the stability of the nozzle flow in dependence on the nozzle pressure ratio and Mach number. Table 1 summarizes the free-stream conditions of the PIV experiments.

Figure 14 shows examples of the recorded PIV images together with the computed instantaneous velocity fields for different flow conditions. The particle image diameter in the raw images was found to be between 3 and 4 pixel. The particle image density of the outer flow varied with the Mach number, respectively, with the mass flow. While the particle image density was about 0.1 particle images per pixel for small Mach numbers, there were often areas with very low seeding densities in the transonic and supersonic range. Only very few particles could be detected within the recirculation region of the step. This indicates that only the largest particles could be seen with the chosen laser and camera set-up, because in earlier works, much more seeding was present in this region $[8,35]$. For the densely seeded PIV images, a background noise level with a standard deviation $\approx 6.8$ counts was estimated from the auto-correlation function with the method presented in [32]. This noise level leads to a loss-of-correlation due to image noise of $F_{\sigma} \approx 0.9$ and to a signal-to-noise ratio of $\mathrm{SNR} \approx 3.5$ based on the findings in [32], which is considered to be well suited for PIV evaluation. However, for regions with reduced particle image density, SNR decreased significantly. As a consequence, many false velocity vectors are predicted in such areas, as can be 

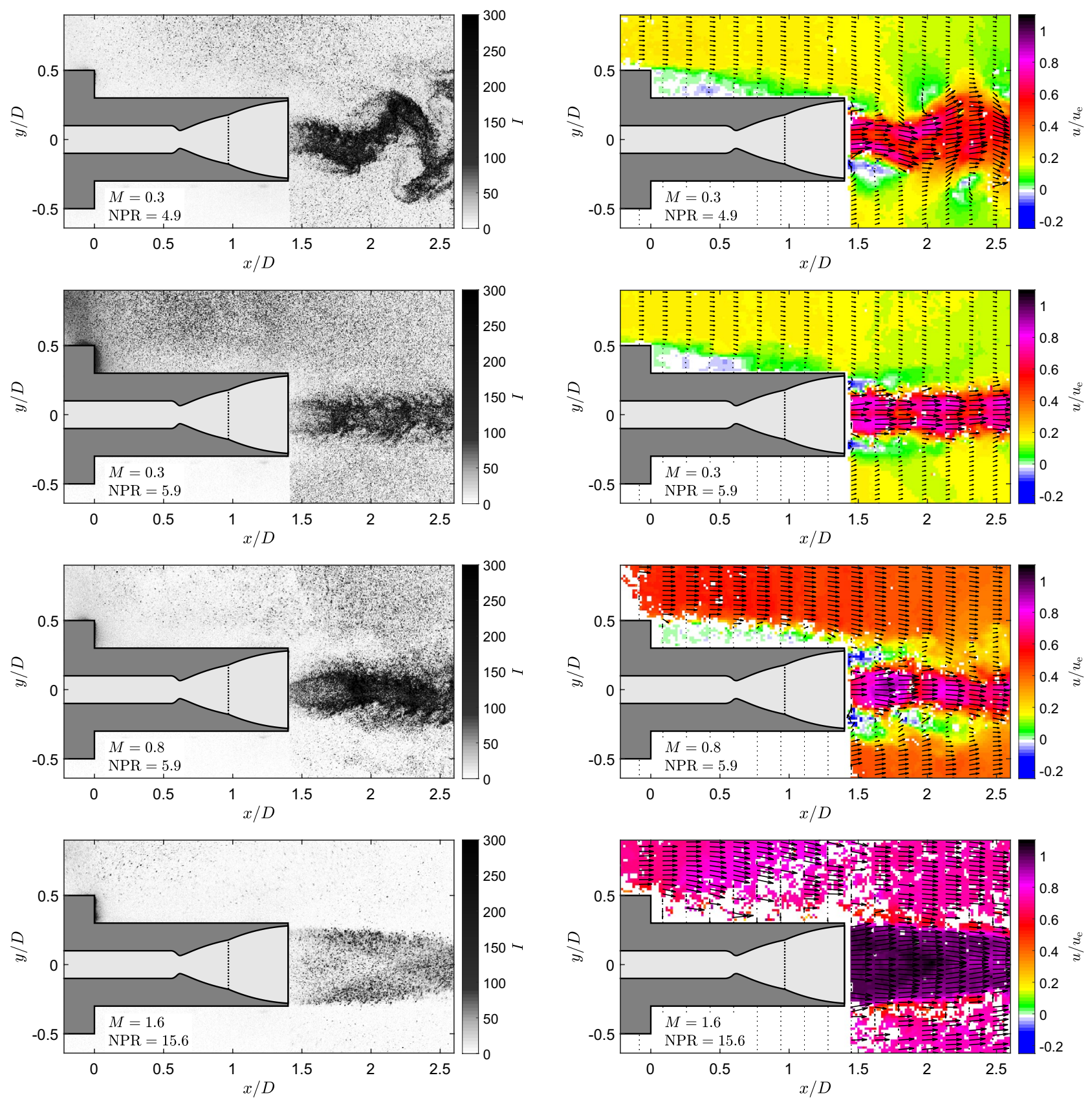

Fig. 14 PIV raw images and corresponding velocity fields for different mach numbers and nozzle pressure ratios. Every 10 th and 2 nd vector is shown in $x$-direction and $y$-direction, respectively

seen from the example velocity fields in the lower 2 rows of Fig. 14.

Although, the optical magnification and the particle image displacement were optimized to account for the richness of spatial and temporal dynamics in this kind of flow, resolving the small-scale features remains challenging due to the strong velocity gradients in the shear layers [33]. The right column of Fig. 14 shows that reliable instantaneous velocity fields could be computed for low subsonic
Mach numbers, while for larger $M$ the reduced seeding density causes invalid vectors, which were attempted to be removed with the method of [58]. To overcome the issues regarding the particle image density and the spatial gradients, single-pixel ensemble correlation was used to compute the mean velocity fields [22, 59]. Additionally, the velocity fluctuations were estimated from the shape of the cross-correlation functions with the method described in [34] including symmetric double correlation [1]. The 

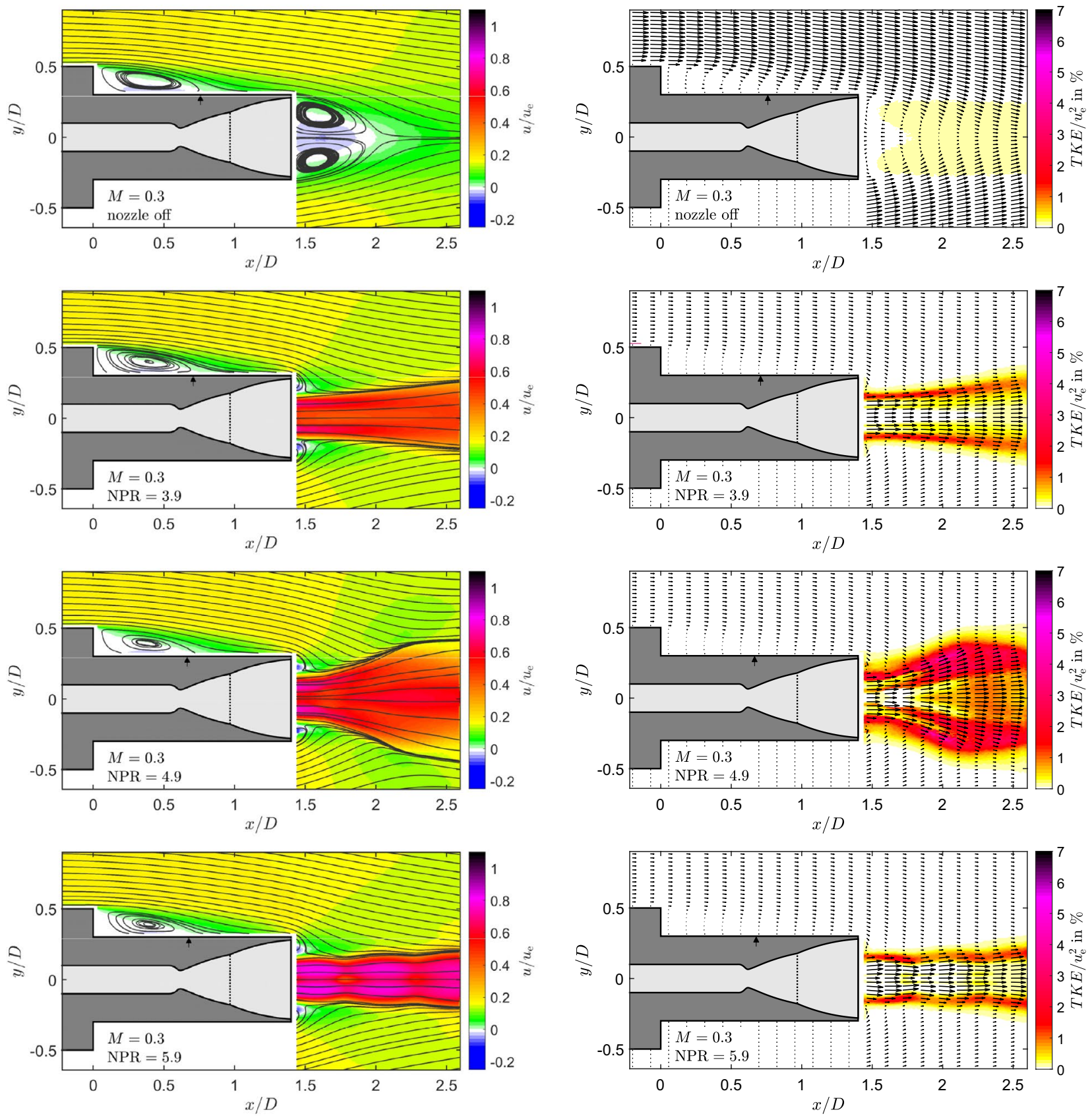

Fig. 15 Mean velocity distribution (left) and turbulent kinetic energy (right) at different nozzle pressure ratios NPR for $M=0.3$. The mean reattachment location is marked by the arrow

single-pixel approach allows for accurate estimation of the flow statistics from a large number of PIV recordings even for cases with varying seeding density. However, in the recirculation area after the step not enough seeding can be seen in the case of the supersonic flow. Consequently, no reliable results can be determined for this area. For the flow statistics, 10,000 PIV double images were recorded per case at a rate of $5 \mathrm{kHz}$, leading to a flow through time of at least 8000 , based in the free-stream velocity $u_{\infty}$ and the model thickness $D$. The time separation between the double images was set to $1.5 \mu \mathrm{s}$ for all measurements, resulting in a displacement of 5 pixel for the free-stream of the outer flow at $M=0.3$ and of 27 pixel for the nozzle flow in altitude mode.

Figures 15,16 and 17 show the mean velocity distribution as well as the turbulent kinetic energy TKE, for $M=0.3,0.8$ and 1.6 at different NPR levels. The two-component TKE is 

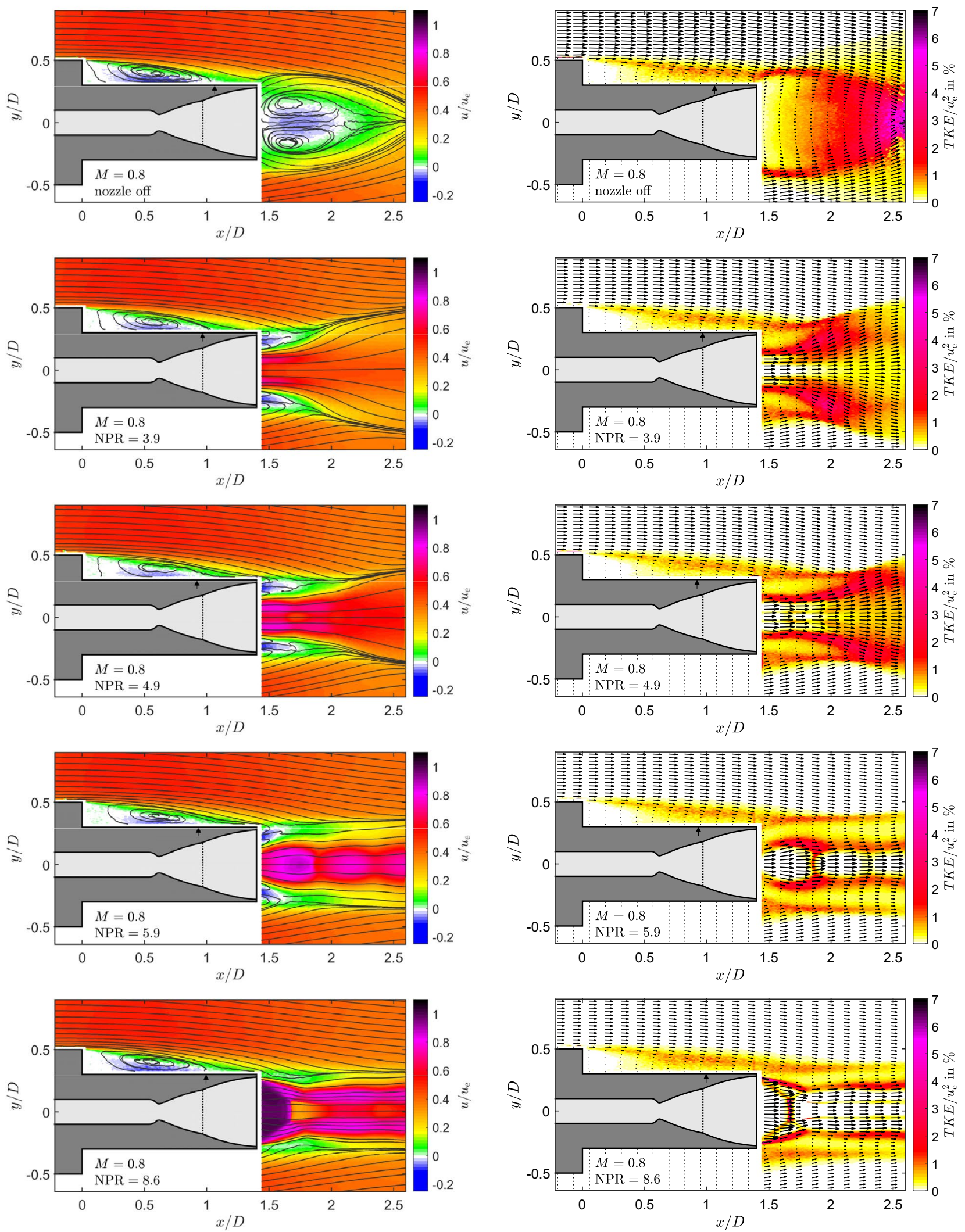

Fig. 16 Mean velocity distribution (left) and turbulent kinetic energy (right) at different nozzle pressure ratios NPR for $M=0.8$. The mean reattachment location is marked by the arrow 

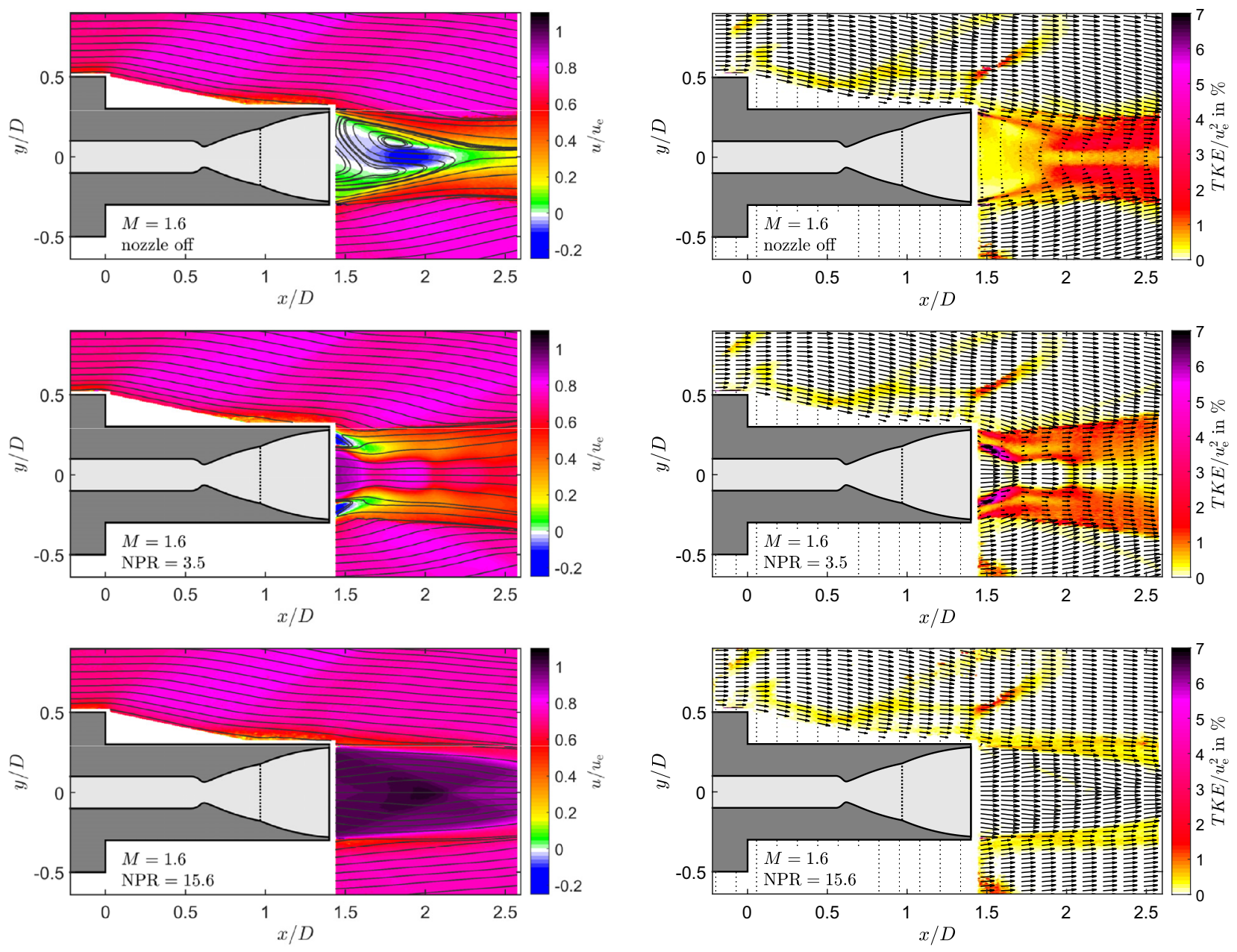

Fig. 17 Mean velocity distribution (left) and turbulent kinetic energy (right) at different nozzle pressure ratios NPR for $M=1.6$. The region downstream of the step is masked out because of noisy data due to low seeding concentration

computed from the standard deviation of the velocity components as follows:

$\mathrm{TKE}=\frac{\sigma_{\mathrm{u}}^{2}+\sigma_{\mathrm{v}}^{2}}{2}$

In the figures, the mean velocity and TKE are normalized with the nozzle exit velocity at altitude mode $u_{\mathrm{e}}=585 \mathrm{~m} / \mathrm{s}$. The single-pixel correlation functions were averaged over $3 \times 3$ pixel and flow statistics are presented for every second pixel in both directions. The resulting vector grid spacing is about $71 \mu \mathrm{m}$ (or $0.3 \%$ of the main body thickness). Every 45th and 10th (in $x$ - and $y$-direction) velocity vector is shown on top of the TKE distribution in the figures.

For all cases shown in Figs. 15, 16 and 17, the flow fields for the different nozzle pressure ratios appear similar for the region upstream of the nozzle exit. At the end of the main body, at $x / D=0$, the incoming boundary layer separates. The free shear layer broadens further downstream and reattaches on the nozzle fairing. The size of recirculation region is characterized by the mean reattachment length, which is indicated by the black arrow. Due to the small number of particle images, the mean reattachment length for the supersonic cases could not be reliably determined and is, therefore, not indicated. For the subsonic cases without nozzle flow, the reattachment length (normalized with the step height) is about $10 \%--15 \%$ shorter compared to the measurements in [8], which were performed on a model with longer splitter plate and without nozzle. On the other hand, numerical simulations of a planar launcher model with dual-bell nozzle in sea-level mode performed by [24] predict a $20 \%$ longer reattachment length for $M=0.8$. Additionally, the average reattachment length decreases slightly with increasing NPR for the subsonic Mach numbers. For $M=0.3$, the mean reattachment location is at $x / D=0.75$ corresponding to 3.75 step heights without the nozzle flow. When the nozzle is switched on, the reattachment point moves further towards the step and for NPR $=6$ it reaches 


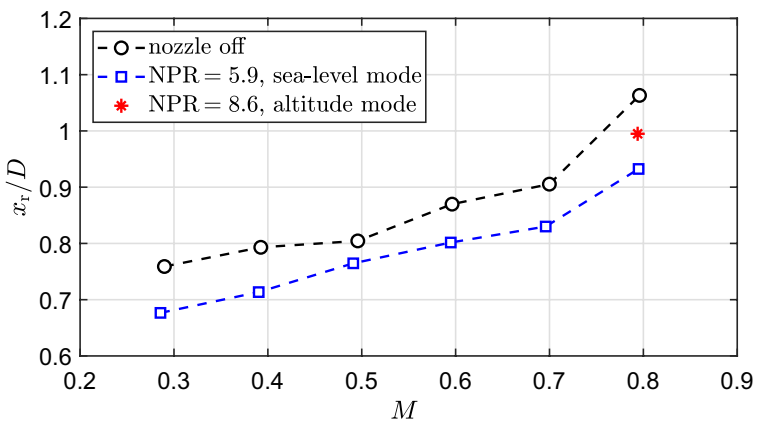

Fig. 18 Mean reattachment length on the nozzle fairing for different subsonic Mach numbers for the case with the nozzle turned off and for $\mathrm{NPR}=5.9$

$x / D=0.68$. For $M=0.8$, the reattachment length is $x / D=1.07$ (corresponding to 5.35 step heights) without the nozzle flow and decreases with increasing nozzle pressure ratio, as before. This trend is observed for all subsonic Mach numbers, as illustrated in Fig. 18. It is interesting to note that the reattachment location moves again downstream when the dual-bell nozzle is operated in altitude mode (see Fig. 16 on the bottom row). In contrast to the presented findings herein, van Gent et al. [15] found that the nozzle flow does not affect the reattachment length in the case of an axisymmetric model with conical nozzle operated at over-expanded condition. It seems likely that the ratio of jet height to nozzle fairing thickness affects the reattachment location. This ratio is relatively small in the case of a dual-bell nozzle operated in sea-level mode, leading to recirculation regions at the nozzle's lip.

Downstream of the nozzle exit, the nozzle flow strongly affects the flow field. With the jet turned off, a large recirculation region forms downstream of the model, refer to the first rows in Figs. 15, 16 and 17. With the nozzle operated in sea-level mode, two smaller recirculation regions develop to the side of the jet. For $M=0.3$, these back-flow regions are significantly smaller than for $M=0.8$ or 1.6 . The subsonic cases show at NPR values of 3.9 and 4.9 a large widening of the jet in the mean velocity distribution as well as high intensities in the TKE distribution. This is caused by strong jet oscillations, as can be seen in the top row of Fig. 14 and Fig. 4. At NPR= 5.9 the nozzle flow is in stable sea-level mode with small regions of elevated TKE values for $M=0.3$ and 0.8 . The individual shock cells can now be clearly identified. At NPR $=8.8$ and 15.6, the dual-bell nozzle is operated in altitude mode at $M=0.8$ and 1.6, respectively. The jet is stable and the TKE values are also low in this case. Furthermore, a recirculation region next to the jet is not visible anymore.

With supersonic outer flow, the pressure felt by the jet is significantly reduced due to Prandtl-Meyer expansions around the nozzle's lip. As a result, the transition from
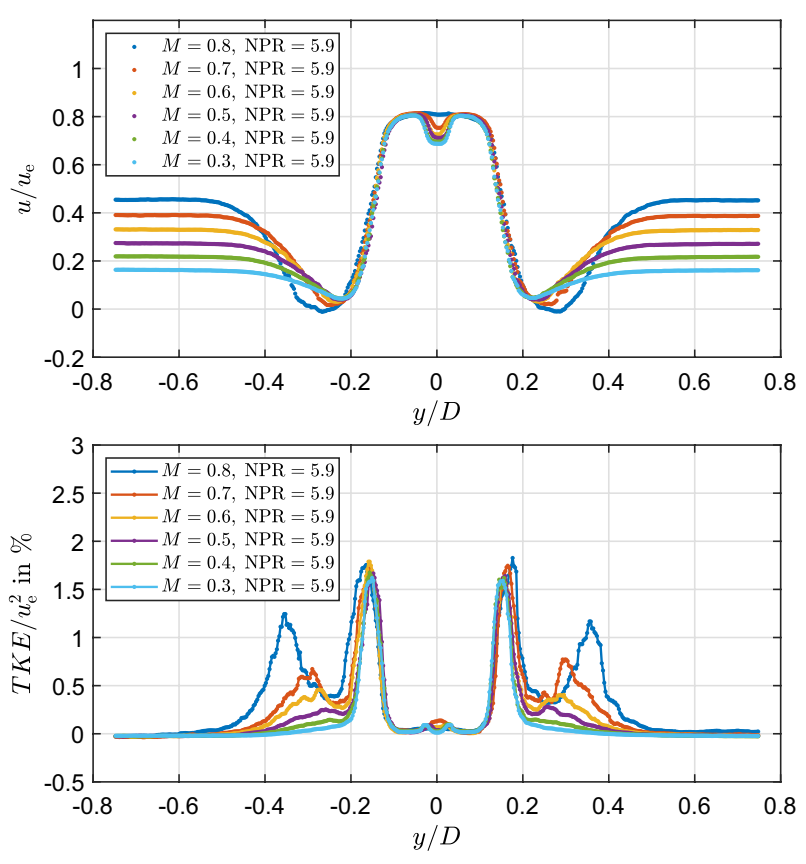

Fig. 19 Profiles of the mean velocity (top) and TKE (bottom) at $x / d=1.55$ for different subsonic Mach numbers at NPR= 5.9

sea-level mode to altitude mode happens at massively reduced NPR compared to the case without outer flow or with subsonic outer flow. Furthermore, the interaction between the jet and the Prandtl-Meyer expansions results in a so-called flip-flop behavior that causes multiple transitions between the two nozzle modes [2,9].

Regarding the nozzle flow state, it is important to note that the nozzle pressure ratio computed from Eq. (1) is not sufficient to predict the nozzle mode, since it is based on the free-stream pressure of the outer flow. However, the pressure in the direct vicinity of the nozzle outlet determines the behavior of the jet. Figure 19 compares profiles of the mean velocity and the turbulent kinetic energy just downstream of the nozzle for different free-stream Mach numbers and a fixed nozzle pressure ration of NPR $=5.9$. It can be seen from the figure, that the nozzle flow is rather independent of $M$. The over-expansion shocks emerging from the contour deflection interact with a Mach reflection for Mach numbers below 0.8 . This results in a local minimum of the velocity in the jet core at $x / d=1.55$. With increasing Mach numbers, the reattachment length grows (see Fig. 18), resulting in increased momentum loss near the nozzle fairing at the end of the model. This increases the static pressure and causes the over-expansion shocks in the jet to become stronger. The stronger shocks for $M=0.8$ perform a regular reflection instead of a Mach reflection, which results in a top-hat like velocity profile. The TKE distribution in Fig. 19 shows that the strongest fluctuations move outwards with increasing Mach number at $x / d=1.55$. This is in agreement with 
the later reattachment that pushes the shear layer further out at $x / d=1.55$.

\section{Summary and Conclusions}

The interaction of the base flow of a generic space launcher model and a cold exhaust jet from a 2D dual-bell nozzle was investigated in this work. The Mach number of the outer flow and the total pressure of the nozzle flow were varied over a large range. The dual-bell nozzle was operated in sea-level mode and in altitude mode. Although the mode transition nozzle pressure ratio was designed to be around $\mathrm{NPR}=10$, it was shown that with outer flow the mode transition already happened at NPR $=8.5$ or 3.5 with a transonic or supersonic outer flow, respectively. This trend was also found for axisymmetric models with dual-bell nozzle [2]. Also truncated plug nozzles show a strong dependency on the Mach number of the outer flow [25]. Since the exact determination of the transition between the modes is essential for planning a mission, it is necessary to investigate the dual-bell nozzle flow together with the external flow. The strong mutual influence is a significant difference to investigations of launcher models with bell-shaped nozzle types [15].

Schlieren measurements with and without jet at a recording frequency of $40 \mathrm{kHz}$ enabled the temporal evolution as well as the statistical analysis of the position of shear layers, shocks and expansions to be recorded. The mean schlieren images in Figs. 5, 6 and 7 visualize the state of the outer flow and the nozzle flow and the corresponding standard deviations capture regions with strong fluctuations. It was shown that for $M<0.7$, the PSD of the schlieren intensity in the outer flow forms additional peaks with the nozzle flow turned on, see Fig. 8. For NPR= 5.0, the exhaust jet performs strong oscillations that lead to a broad region of high PSD values for a frequency of $13.0 \mathrm{kHz}$, as shown in Figs. 9 and 10. The strongest effect on the separated shear layer was found for NPR=7.0 at $M=0.3$ and NPR=7.7 at $M=0.5$. For $M \geq 0.7$, the interaction between separated shear layer and nozzle flow is substantially lower.

The mean velocity fields and the TKE destributions in Figs. 15, 16 and 17 supplement the schlieren recordings. They provide additional information regarding the size of the recirculation region downstream of the step and near the nozzles lip in sea-level mode. It was shown that the mean reattachment length increases with increasing Mach number in the subsonic regime. Furthermore, it was shown that it is about $10 \%$ lower with the nozzle turned on at NPR=5.9. Regarding the nozzle flow, it was shown that the velocity profile at the nozzle exit is affected by the Mach number, respectively, by the reattachment length. For a fixed value of $\mathrm{NPR}=5.9$, the velocity profile features a local minimum in the jet's core for $M \leq 0.7$ that disappears for $M=0.8$, indicating a transition from a Mach reflection to a regular reflection of the over-expansion shocks emerging from the contour junction.

It can be concluded that the nozzle flow influences the separated shear layer and vice versa. The mean reattachment length, the strength of the nozzle shocks and even the dual-bell nozzle mode depend on both, Mach number of the outer flow and nozzle pressure ratio. Thus, it is important to investigate both phenomena together to develop future space launcher propulsion systems. However, this is quite complicated, which is why the experiments described could only be carried out with a cold jet and a two-dimensional model. Further investigations with realistic models and hot plume are essential to verify the observed sensitivities.

Acknowledgements Financial support from the German Research Foundation DFG in the framework of the SFB-TRR40 is gratefully acknowledged. Furthermore, the authors like to thank Chloé Génin for providing the nozzle contour, Istvan Bolgar for his contributions to the project and Matthew Bross for his careful proofreading of the manuscript.

Funding Open Access funding provided by Projekt DEAL.

Open Access This article is licensed under a Creative Commons Attribution 4.0 International License, which permits use, sharing, adaptation, distribution and reproduction in any medium or format, as long as you give appropriate credit to the original author(s) and the source, provide a link to the Creative Commons licence, and indicate if changes were made. The images or other third party material in this article are included in the article's Creative Commons licence, unless indicated otherwise in a credit line to the material. If material is not included in the article's Creative Commons licence and your intended use is not permitted by statutory regulation or exceeds the permitted use, you will need to obtain permission directly from the copyright holder. To view a copy of this licence, visit http://creativecommons.org/licenses/by/4.0/.

\section{References}

1. Avallone, F., Discetti, S., Astarita, T., Cardone, G.: Convergence enhancement of single-pixel PIV with symmetric double correlation. Exp. Fluids 56(4), 1-11 (2015). https://doi.org/10.1007/ s00348-015-1938-2

2. Barklage, A., Radespiel, R., Genin, C.: Afterbody jet interaction of a dual-bell nozzle in supersonic flow. In: 2018 Joint Propulsion Conference, p 4468. (2018)

3. Barklage, A., Loosen, S., Schröder, W., Radespiel, R.: Reynolds number influence on the hysteresis behavior of a dual-bell nozzle. In: 8th European Conference for Aeronautics and Space Science (EUCASS), Madrid, Spain, July 1-4. (2019)

4. Bitter, M., Scharnowski, S., Hain, R., Kähler, C.J.: High-repetition-rate PIV investigations on a generic rocket model in sub- and supersonic flows. Exp. Fluids 50, 1019-1030 (2011). https://doi. org/10.1007/s00348-010-0988-8

5. Bitter, M., Hara, R., Asai, K., Kähler, C.J.: Characterization of pressure dynamics in an axisymmetric separating/reattaching flow 
using fast-responding pressure-sensitive paint. Exp. Fluids 53, 1737-1749 (2012). https://doi.org/10.1007/s00348-012-1380-7

6. Bolgar, I., Scharnowski, S., Kähler, CJ.: Control of the reattachment length of a transonic 2D backward-facing step flow. In: Proceedings of the 5th International Conference on Jets, Wakes and Separated Flows (ICJWSF2015), Springer, pp 241-248. (2016). https://doi.org/10.1007/978-3-319-30602-5_30

7. Bolgar, I., Scharnowski, S., Kähler, C.J.: Passive flow control for the reduction of load dynamics aft of a backward-facing step. AIAA J. 57(1), 120-131 (2018a). https://doi.org/10.2514/1.J0572 74

8. Bolgar, I., Scharnowski, S., Kähler, C.J.: The effect of the Mach number on a turbulent backward-facing step flow. Flow Turbul. Combust. 101, 653-680 (2018b). https://doi.org/10.1007/s1049 4-018-9921-7

9. Bolgar, I., Scharnowski, S., Kähler, C.J.: Experimental Analysis of the Interaction Between a Dual-Bell Nozzle with an External Flow Field Aft of a Backward-Facing Step. In: Dillmann, A., Heller, G., Krämer, E., Wagner, C., Tropea, C., Jakirlić, S. (eds.) New Results in Numerical and Experimental Fluid Mechanics XII, pp. 405-415. Springer International Publishing, Cham (2020). https ://doi.org/10.1007/978-3-030-25253-3_39

10. Deck, S., Thorigny, P.: Unsteadiness of an axisymmetric separating-reattaching flow: Numerical investigation. Phys. Fluids 19, 065103 (2007). https://doi.org/10.1063/1.2734996

11. Depres, D., Reijasse, P., Dussauge, J.: Analysis of Unsteadiness in Afterbody Transonic Flows. AIAA J. 42, 2541-2550 (2004). https://doi.org/10.2514/1.7000

12. Foster, C.R., Cowles, F.B.: Experimental study of gas-flow separation in overexpanded exhaust nozzles for rocket motors. Tech. Rep. 4-103, Jet Propulsion Laboraty, California Institute of Technology, (1949). https://ntrs.nasa.gov/search.jsp?R=19630039654

13. Frey, M., Hagemann, G.: Critical assessment of dual-bell nozzles. J. Propuls. Power 15(1), 137-143 (1999). https://doi. org $/ 10.2514 / 2.5402$

14. Génin, C.: Experimental study of flow behaviour and thermal loads in dual bell nozzles. PhD thesis, Valenciennes (2010)

15. van Gent, P.L., Payanda, Q., Brust, S.G., van Oudheusden, B.W., Schrijer, F.F.J.: Effects of Exhaust Plume and Nozzle Length on Compressible Base Flows. AIAA J. 57(3), 1184-1199 (2019). https://doi.org/10.2514/1.j057314

16. Hagemann, G., Immich, H., Van Nguyen, T., Dumnov, G.E.: Advanced rocket nozzles. J. Propuls. Power 14(5), 620-634 (1998). https://doi.org/10.2514/2.5354

17. Hampel, A.J.: Auslegung, Optimierung und Erprobung eines vollautomatisch arbeitenden Transsonik-Windkanals. PhD thesis, Bundeswehr University Munich (1984)

18. Hannemann, K., Lüdeke, H., Pallegoix, JF., Ollivier, A., Lambaré, H., Maseland, J., Geurts, E., Frey, M., Deck, S., Schrijer, F., Scarano, F., Schwane, R.: Launcher vehicle base buffeting - recent experimental and numerical investigations. In: Proceedings 7th European Symposium on Aerothermodynamics for Space Vehicles, Brugge, Belgium, 9-12 May (2011)

19. Hargather, M.J., Settles, G.S.: A comparison of three quantitative schlieren techniques. Opt. Lasers Eng. 50(1), 8-17 (2012). https ://doi.org/10.1016/j.optlaseng.2011.05.012

20. Horn, M., Fisher, S.: Dual-bell altitude compensating nozzles, Pennsylvania State Univ., NASA Propulsion Engineering Research Center, Volume 2 (1993). https://ntrs.nasa.gov/citat ions/19940018584

21. Kähler, C.J., Sammler, B., Kompenhans, J.: Generation and control of particle size distributions for optical velocity measurement techniques in fluid mechanics. Exp. Fluids 33, 736-742 (2002). https://doi.org/10.1007/s00348-002-0492-x
22. Kähler, C.J., Scharnowski, S., Cierpka, C.: On the resolution limit of digital particle image velocimetry. Exp. Fluids 52, 1629-1639 (2012). https://doi.org/10.1007/s00348-012-1280-x

23. Loosen, S., Statnikov, V., Meinke, M., Schröder, W.: Modal analysis of passive flow control for the turbulent wake of a generic planar space launcher. CEAS Space J. 10(2), 189-202 (2018). https://doi.org/10.1007/s12567-017-0183-3

24. Loosen, S., Meinke, M., Schröder, W.: Numerical Investigation of Jet-Wake Interaction for a Dual-Bell Nozzle. Flow Turbul. Combust. 104(2), 553-578 (2020). https://doi.org/10.1007/s1049 4-019-00056-6

25. Nasuti, F., Onofri, M.: Analysis of in-flight behavior of truncated plug nozzles. J. Propuls. Power 17(4), 809-817 (2001). https:// arc.aiaa.org/doi/pdfplus/10.2514/2.5837

26. Nasuti, F., Onofri, M., Martelli, E.: Role of wall shape on the transition in axisymmetric dual-bell nozzles. J. Propuls. Power 21(2), 243-250 (2005). https://doi.org/10.2514/1.6524

27. Nürnberger-Genin, C., Stark, R.: Flow transition in dual bell nozzles. Shock Waves 19(3), 265 (2009). https://doi.org/10.1007/ s00193-008-0176-4

28. Pain, R., Weiss, P.E., Deck, S.: Zonal Detached Eddy Simulation of the flow around a simplified launcher afterbody. AIAA J. 52(9), 1967-1979 (2014). https://doi.org/10.2514/1.J052743

29. Ragni, D., Schrijer, F., Van Oudheusden, B.W., Scarano, F.: Particle tracer response across shocks measured by PIV. Exp. Fluids 50(1), 53-64 (2011). https://doi.org/10.1007/s00348-010-0892-2

30. Raman, G.: Supersonic jet screech: half-century from Powell to the present. J. Sound Vib. 225(3), 543-571 (1999). https://doi. org/10.1006/jsvi.1999.2181

31. Saile, D., Kühl, V., Gülhan, A.: On the subsonic near-wake of a space launcher configuration without jet. Exp. Fluids 60(4), 50 (2019). https://doi.org/10.1007/s00348-019-2690-9

32. Scharnowski, S., Kähler, C.J.: On the loss-of-correlation due to PIV image noise. Exp. Fluids 57(7), 1-12 (2016). https://doi. org/10.1007/s00348-016-2203-Z

33. Scharnowski, S., Kähler, CJ.: Particle Image Velocimetry - classical operating rules from today's perspective. Opt. Lasers Eng. 106185, (2020) https://doi.org/10.1016/j.optlaseng.2020.106185

34. Scharnowski, S., Hain, R., Kähler, C.J.: Reynolds stress estimation up to single-pixel resolution using PIV-measurements. Exp. Fluids 52, 985-1002 (2012). https://doi.org/10.1007/s00348-011-1184-1

35. Scharnowski, S., Bolgar, I., Kähler, C.J.: Characterization of Turbulent Structures in a Transonic Backward-Facing Step Flow. Flow Turbul. Combust. 98(4), 947-967 (2017). https://doi. org/10.1007/s10494-016-9792-8

36. Scharnowski, S., Bosyk, M., Schrijer, F.F.J., van Oudheusden, B.W.: Passive Flow Control for the Load Reduction of Transonic Launcher Afterbodies. AIAA J. 57(5), 1818-1825 (2019a). https ://doi.org/10.2514/1.j057357

37. Scharnowski, S., Bross, M., Kähler, C.J.: Accurate turbulence level estimations using PIV/PTV. Exp. Fluids 60(1), 1 (2019b). https://doi.org/10.1007/s00348-018-2646-5

38. Scheitle, H., Wagner, S.: A four colours line grid schlieren method for quantitative flow measurement. Exp. Fluids 9(6), 333-336 (1990). https://doi.org/10.1007/bf00188763

39. Scheitle, H., Wagner, S.: Influences of wind tunnel parameters on airfoil characteristics at high subsonic speeds. Exp. Fluids 12(1-2), 90-96 (1991). https://doi.org/10.1007/BF00226571

40. Schneider, D., Génin, C.: Numerical investigation of flow transition behavior in cold flow dual-bell rocket nozzles. J. Propuls. Power pp 1212-1219 (2016). https://doi.org/10.2514/1.B36010

41. Schneider, D., Stark, R.H., Génin, C., Oschwald, M., Kostyrkin, K.: Operation Mode Transition of Film-Cooled Dual-Bell Nozzles. In: 2018 Joint Propulsion Conference, p 4467, (2018). https ://doi.org/10.2514/6.2018-4467 
42. Schreyer, A-M.: Flow structure in the wake of a space-launcher model with propulsive-jet simulation. CEAS Space J. pp 1-17, (2020). https://doi.org/10.1007/s12567-020-00306-8

43. Schrijer, F.F.J., Sciacchitano, A., Scarano, F.: Spatio-temporal and modal analysis of unsteady fluctuations in a high-subsonic base flow. Phys. Fluids 26(8), 086101 (2014). https://doi. org/10.1063/1.4891257

44. Settles, G.S.: Schlieren and shadowgraph techniques: visualizing phenomena in transparent media. Springer-Verlag, Berlin Heidelberg (2012). https://doi.org/10.1007/978-3-642-56640-0

45. Stark, R., Génin, C.: Sea-level transitioning dual bell nozzles. CEAS Space J. 9(3), 279-287 (2017). https://doi.org/10.1007/ s12567-017-0154-8

46. Stark, R., Génin, C., Schneider, D., Fromm, C.: Ariane 5 Performance Optimization Using Dual-Bell Nozzle Extension. J. Spacecr. Rockets 53(4), 743-750 (2016). https://doi. org/10.2514/1.a33363

47. Statnikov, V., Bolgar, I., Scharnowski, S., Meinke, M., Kähler, C.J., Schröder, W.: Analysis of characteristic wake flow modes on a generic transonic backward-facing step configuration. Eur. J. Mech.-B/Fluids 59, 124-134 (2016). https://doi.org/10.1016/j. euromechflu.2016.05.008

48. Statnikov, V., Meinke, M., Schröder, W.: Reduced-order analysis of buffet flow of space launchers. J. Fluid Mech. 815, 1-25 (2017). https://doi.org/10.1017/jfm.2017.46

49. Stephan, S., Wu, J., Radespiel, R.: Propulsive jet influence on generic launcher base flow. CEAS Space J. 7(4), 453-473 (2015). https://doi.org/10.1007/s12567-015-0098-9

50. Takahashi, H., Tomita, T., Génin, C., Schneider, D.: LOX/CH 4 Hot-Flow Testing of Dual-Bell Nozzles with Rapid-Expansion Extension. J. Propuls. Power 34(5), 1214-1224 (2018). https:// doi.org/10.2514/1.b36768

51. Verma, M., Arya, N., De, A.: Investigation of flow characteristics inside a dual bell nozzle with and without film cooling. Aerosp. Sci. Technol. 99, 105741 (2020). https://doi.org/10.1016/j. ast.2020.105741
52. Verma, S.B., Stark, R., Nuerenberger-Genin, C., Haidn, O.: Coldgas experiments to study the flow separation characteristics of a dual-bell nozzle during its transition modes. Shock Waves 20(3), 191-203 (2010). https://doi.org/10.1007/s00193-010-0259-x

53. Verma, S.B., Stark, R., Haidn, O.: Effect of ambient pressure fluctuations on dual-bell transition behavior. J. Propuls. Power 30(5), 1192-1198 (2014). https://doi.org/10.2514/1.b35067

54. Weiss, P.É., Deck, S.: Control of the antisymmetric mode $(\mathrm{m}=$ 1) for high Reynolds axisymmetric turbulent separating/reattaching flows. Phys. Fluids 23(9), 095102 (2011). https://doi. org/10.1063/1.3614481

55. Weiss, P.É., Deck, S.: Numerical investigation of the robustness of an axisymmetric separating/reattaching flow to an external perturbation using ZDES. Flow Turbul. Combust. 91(3), 697-715 (2013). https://doi.org/10.1007/s10494-013-9484-6

56. Weiss, P.É., Deck, S., Robinet, J.C., Sagaut, P.: On the dynamics of axisymmetric turbulent separating/reattaching flows. Phys. Fluids 21, 075103 (2009). https://doi.org/10.1063/1.3177352

57. Welch, P.D.: he Use of Fast Fourier Transform for the Estimation of Power Spectra: A Method Based on Time Averaging Over Short, Modified Periodograms. IEEE Trans. Audio Electroacoust. AU- 15, 70-73 (1967). https://doi.org/10.1109/TAU.1967.11619 01

58. Westerweel, J., Scarano, F.: Universal outlier detection for PIV data. Exp. Fluids 39, 1096-1100 (2005). https://doi.org/10.1007/ s00348-005-0016-6

59. Westerweel, J., Geelhoed, P.F., Lindken, R.: Single-pixel resolution ensemble correlation for micro-PIV applications. Exp. Fluids 37, 375-384 (2004). https://doi.org/10.1007/s00348-004-0826-y

Publisher's Note Springer Nature remains neutral with regard to jurisdictional claims in published maps and institutional affiliations. 